\title{
Controlling Particle Size and Release Kinetics in the Sustained Delivery of Oral Antibiotics Using pH-Independent Mucoadhesive Polymers
}

\author{
Cristina Yus, Silvia Irusta, Victor Sebastian,* and Manuel Arruebo*
}

Cite This: https://dx.doi.org/10.1021/acs.molpharmaceut.0c00408

Read Online

ACCESS | Llll Metrics \& More | 国 Article Recommendations | sl Supporting Information

\begin{abstract}
Controlling antibiotic delivery using pH-mucoadhesive polymeric $\mu /$ nano particles
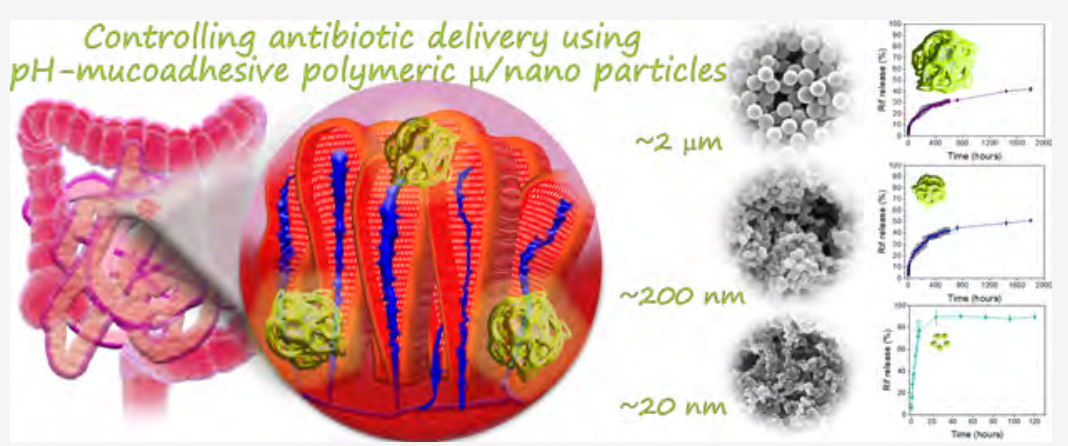

ABSTRACT: Copolymers synthesized from acrylic acid and methacrylic acid used as gastroprotective and mucoadhesive enteric coatings have been used to prepare micro- $(\sim 2 \mu \mathrm{m})$, submicro- $(\sim 200 \mathrm{~nm})$, and nanoparticles $(\sim 20 \mathrm{~nm})$ containing rifampicin (Rif) to obtain time-controlled drug release kinetics. Different particle sizes and drug release kinetics have been obtained using different synthesis conditions and fabrication techniques including the use of an electrosprayer and an interdigital microfabricated micromixer. The antimicrobial action of the encapsulated Rif has been demonstrated against Staphylococcus aureus ATCC 25923 and compared with the effect of the equivalent dose of the free macrolide antibiotic. At low concentrations, the encapsulated antibiotic showed superior antimicrobial activity than the free drug. The stability of the developed particles has been evaluated in vitro under simulated gastric and intestinal conditions. At the concentrations tested, a reduced cytotoxicity against different human cell lines was observed after analyzing their subcytotoxic doses and the influence on their cell cycle by flow cytometry. Drug release kinetics can be tuned by adjusting particle sizes, and it would be possible to reach the minimum inhibitory concentration or the minimum bactericidal concentration at different time points depending on the medical needs.
\end{abstract}

KEYWORDS: Eudragit, drug delivery, size-dependent release kinetics, Staphylococcus aureus, antimicrobial activity, rifampicin, cytocompatibility

\section{INTRODUCTION}

Antimicrobial delivery systems have been developed to provide therapeutic doses at predetermined rates above the minimum bactericidal concentration (MBC) and below the maximum toxicological threshold. Extended-release dosage forms maintain sufficient antibiotic plasma concentration and promote patient compliance, improving the therapeutic index. Furthermore, antibiotic encapsulation in biodegradable matrices prolong the duration of hydrophobic biocides, while reducing unwanted side effects. In this regard, Sung et al. ${ }^{1}$ demonstrated, in preclinical models, that the encapsulation of rifampicin (Rif), one of the first-line antituberculotic antibiotic, within poly(lactic-co-glycolic acid) (PLGA) nanoparticles provided a prolonged presence in the lungs. A sevenfold dose sparing was demonstrated by Lueth et al. ${ }^{2}$ when comparing the bacterial counts present in Brucella melitensis-infected mice from animals treated with a weekly dose of doxycycline-encapsulated nanoparticles and with the daily dose of the free drug.

Antibiotic-associated adverse side effects might include hemolytic activity and cytotoxicity against mammalian cells. However, the encapsulation of several antimicrobials within nanoparticulated carriers can alleviate these side effects. In this regard, Li et al. ${ }^{3}$ confirmed that the cytotoxicity of ciprofloxacin against HeLa cells and the hemolytic activity of an antimicrobial copolymer can be reduced when encapsulated within polymeric nanoparticulated vesicles. Hussain et al. ${ }^{4}$

Received: April 16, 2020

Revised: July 17, 2020

Accepted: July 20, 2020

Published: July 20, 2020 
demonstrated not only that nanoencapsulated vancomycin was 10-fold more effective than free vancomycin in a Staphylococcus aureus-induced pneumonia model in vivo but also they proved the lack of toxicity to the liver. Reduced cytotoxicity against mammalian cells has also been reported when conjugating Rif to nanoparticulated carriers in the eradication of intracellular pathogens (Mycobacterium tuberculosis) in coculture models. ${ }^{5}$

Encapsulation can also provide antibiotics with enhanced bioavailability and protection from physiological degradation. In this regard, increased skin penetration, reduced haemolytic activity, and a lack of cytotoxicity against mammalian cell lines were reported in a methicillin-resistant $S$. aureus (MRSA)infected burn-wound animal model when using chloramphenicol-loaded poly(epsilon-caprolactone) (PCL)-Pluronic nanoparticles compared to the administration of the free antibiotic. ${ }^{6}$ Several antibiotics have been gastroprotected to prevent their degradation during the presystemic metabolism, improving their bioavailability. Antibiotic-mixed matrices and amorphous solid dispersions with gastroprotective materials or their encapsulation as thin coatings on the active principle have been included in the formulations to overcome these limitations. For instance, clarithromycin has been protected with carboxyl-containing cellulose derivatives, forming nanoparticles to quench the acid-catalyzed degradation in the stomach and improve the aminomacrolide antibiotic solubility in the intestine. Likewise, cellulose derivatives have also been used to protect rifapentine from gastric degradation and to improve its reduced solubility at intestinal $\mathrm{pH}^{8}$ According to Singh et al., $26.5 \%$ of free Rif was degraded using simulated gastric fluid (SGF) with its degradation aggravated under the presence of one of the secondary antibiotics used (isoniazid) as common combination therapy. ${ }^{9}$ Conversely, its encapsulation in solid lipid nanoparticles prepared by microemulsification has been demonstrated to reduce its degradation by threefold. ${ }^{9}$ Compared to the oral administration of the free drug in preclinical models, improved pharmacokinetics (i.e., area under the concentration-time curve and mean residence time) have also been achieved by encapsulating Rif within gelatin-based nanoparticles. ${ }^{10}$ Not only lipids, polymers, and polysaccharides of natural origin are used to obtain gastroprotection but also synthetic polymers can be used to take advantage of their ability to produce time-controlled drug release to specific anatomical areas in the intestinal lining. Copolymers of methyl acrylate, methyl methacrylate, and methacrylic acid are used to avoid gastric degradation and to achieve $\mathrm{pH}$-dependent or $\mathrm{pH}$ independent intestinal delivery of encapsulated antibiotics. Some of them are $\mathrm{pH}$-dependent and remain protonated and insoluble in the stomach but dissolve in the intestine; others are mucoadhesive in the intestinal lining and being insoluble, they erode over time providing with a time-controlled targeted release of the encapsulated active principle. We have previously encapsulated Rif within $\mathrm{pH}$-dependent methacrylate-based microparticles (i.e., Eudragit L100-55) and demonstrated their antibiotic protection against SGF, being the active antibiotic able to cross an in vitro model of the intestinal barrier. ${ }^{11}$ Compared to the oral administration in human subjects of the free drug and the equivalent dose of Rif encapsulated in Eudragit S100, a superior bioavailability and sustained release were demonstrated for the latter. ${ }^{12}$ The gastroprotection of Rif in these carriers cannot only protect the drug from the acidic oxidative degradation but also improve its bioavailability, overcoming its reduced water solubility (2.5 $\mathrm{mg} / \mathrm{mL}$ at $25{ }^{\circ} \mathrm{C}, \mathrm{pH}$ 7.3). Protecting the cargo against degradation and providing with a timely drug release at a specific anatomical location in the digestive tract would be ideal to maximize active drug absorption.

Rif is an antimycobacterial agent that also inhibits ribonucleic acid synthesis in a wide range of other microbial pathogens. Because of its rapid development of bacterial resistance, it is commonly used in combination with other antimycobacterial agents (isoniazid, pyrazinamide, and ethambutol). Implant-associated infections have also been treated in vivo with Rif as combination therapy in the successful eradication of multidrug-resistant (MDR) Gram-negative bacteria colonizing catheters ${ }^{13}$ and Gram-positive bacteria (i.e., MRSA) colonizing infected coverslips used as models of implant-associated infections. ${ }^{14}$ S. aureus has also been used as a model of pathogen having part of its life cycle intracellularly. In this regard, Sandberg et al. ${ }^{15}$ demonstrated, using a murine peritonitis/sepsis model, that Rif was able to eliminate both intracellular and extracellular bacteria, and compared with other four antibiotics (i.e., dicloxacillin, cefuroxime, gentamicin, and azithromycin), Rif showed the highest antimicrobial effect extracellularly. Three randomized controlled trials and one cohort reported on adults diagnosed with $S$. aureus bacteraemia (SAB) revealed that Rif in combination with standard $\beta$-lactam or glycopeptide therapy might produce an improved clinical outcome in $\mathrm{SAB}$ treatment. ${ }^{16} \mathrm{~S}$. aureusassociated chronic osteomyelitis has also been treated in vivo with Rif because this wide-spectrum antibiotic has been demonstrated to be superior when reducing bacterial loads compared to $\beta$-lactams, fluoroquinolones, vancomycin, linezolid, daptomycin, fosfomycin, gentamicin, and clindamycin. ${ }^{17}$ With S. aureus biofilms formed on implants as a model of osteomyelitis, Jørgensen et al. ${ }^{18}$ also confirmed its superior antimicrobial action in vivo alone or in combination when compared to vancomycin, daptomycin, and linezolid. However, further standardized double-blinded randomized clinical trials are required to justify the use of Rif because adverse drug interactions and the appearance of Rif resistance may overshadow its demonstrated benefits in the treatment of $S$. aureus-associated infections. ${ }^{19}$

Therefore, gastroprotection and targeted delivery to improve absorption and systemic distribution of Rif might improve its pharmacokinetics in the treatment of $S$. aureus-associated infections. In this work, we have used the $\mathrm{pH}$-independent and mucoadhesive Eudragit RS100 (EuRS100) polymer as a drug carrier to encapsulate the antimycobacterial agent Rif. As a novelty, we have demonstrated that particle size and drug loadings can be adjusted by selecting the proper fabrication process. Finally, we have varied the physical properties of the Rif-loaded carriers in order to control drug release kinetics and achieve an effective and timely antimicrobial action to potentially prevent S. aureus (ATCC 25923)-associated infections. In addition, the mucoadhesive character of the carrier on the intestinal lining would allow a sustained release of the antimicrobial cargo over time to provide with a therapeutic dose. This release can be tunable depending on the needs by properly selecting the particle size and structure.

\section{EXPERIMENTAL SECTION}

Materials. EuRS100 was generously donated by Evonik Industries AG (Essen, Germany). Chloroform (anhydrous, $\geq 99 \%$ ), acetone (>99\%), dimethyl sulfoxide (DMSO, > 99\%), Rif ( $\geq 97 \%)$, and phosphate-buffered saline (PBS) were purchased from Sigma-Aldrich (Darmstadt, Germany). Etha- 
nol absolute (EtOH) was purchased from PanReac AppliChem (Barcelona, Spain). All reagents were used as received without any further purification. SGF $\left(\mathrm{HCl} 0.1 \mathrm{~N}, \mathrm{NaCl}\right.$ and $\mathrm{H}_{2} \mathrm{O}, \mathrm{pH}$ $=1.1$ ) without enzymes was prepared according to the US Pharmacopoeia XXIII (1995).

S. aureus (ATCC 25923) was purchased from Ielab (Alicante, Spain). Tryptone soy broth (TSB) and tryptone soy agar were purchased from Laboratorios Conda-Pronadisa SA, (Torrejon de Ardoz, Spain). Caco2-TC7 clone human epithelial colorectal adenocarcinoma cells were kindly donated by Dr. M.J. Rodriguez Yoldi. Human dermal fibroblasts were obtained from Lonza (Bornem, Belgium). High-glucose Dulbecco's modified Eagle's medium (DMEM; DMEM w/ stable glutamine) and antibiotic-antimycotic $(60 \mu \mathrm{g} / \mathrm{mL}$ penicillin, $100 \mu \mathrm{g} / \mathrm{mL}$ streptomycin, and $0.25 \mu \mathrm{g} / \mathrm{mL}$ amphotericin B) were supplied by Biowest (Nuaillé, France), supplemented with $10 \%$ (v/v) fetal bovine serum (FBS) from Gibco (Thermo Fisher Scientific, Waltham, Massachusetts, USA). Blue cell viability assay was purchased from Abnova (Abnova GmbH, Taipei, Taiwan).

Synthesis of Nano- and Microparticles. Depending on the particle size desired, two fabrication techniques were used, namely, electrospraying and nanoprecipitation. The electrosprayed particles were synthesized in a Yflow 2.2.D-500 electrospinner (Electrospinning Machines/R\&D Microencapsulation, Valencia, Spain) by the action of electro-hydrodynamic forces. The initial polymer solutions were composed of $13 \%(\mathrm{w} / \mathrm{v})$ EuRS100 in chloroform and 3\% (w/v) EuRS100 in $\mathrm{EtOH} /$ acetone $(1: 1)$. For Rif encapsulation, an adequate amount of antibiotic [15 and $20 \%(\mathrm{w} / \mathrm{w})$ referred to the amount of EuRS100 added] was added to the precursor polymer solution, and the final solution was sonicated for 10 min. The corresponding Rif-EuRS100 solutions were introduced into a $10 \mathrm{~mL}$ syringe connected to a $22 \mathrm{G}$ needle, and the flow rate was set at $0.5 \mathrm{~mL} / \mathrm{min}$. A flat collector was used to collect the resulting particles, and the distance between the tip and the collector was set at $10 \mathrm{~cm}$. The positive voltage applied to the needle was $6.4 \mathrm{kV}$ for EuRS100 13\% (w/v) and $12.5 \mathrm{kV}$ for EuRS100 3\% (w/v), and the negative voltages applied to the collector were -3.4 and $-4.7 \mathrm{kV}$, respectively. Particles were synthesized at room temperature with a relative humidity between 30 and 50\%. The collector was covered with aluminum foil. After spraying, the produced microparticles were gently scratched from the foil.

Nanoprecipitated particles were obtained in a commercial polyether ether ketone (PEEK)-based interdigital microfluidic device (Micro4 Industries $\mathrm{GmbH}$, Mainz, Germany), described elsewhere. ${ }^{20}$ Polymer solutions of $5 \%(\mathrm{w} / \mathrm{v})$ EuRS100 in EtOH/acetone $(1: 1)$ and $5 \%(\mathrm{w} / \mathrm{w})$ of Rif referred to EuRS100 were used as solvent phases. The antisolvent phase consisted of milliQ water. Both phases were introduced into two syringes, and the total flow rate was set at $5 \mathrm{~mL} / \mathrm{min}$ using syringe pumps (Harvard Apparatus, Holliston, MA, USA), and the flow rate antisolvent/solvent ratio was set at $R=5$. Solvent and antisolvent phases were interfaced in the interdigital micromixer. The nanoprecipitated particles were collected at the outlet of the micromixer and were evaporated at room temperature under magnetic stirring $(600 \mathrm{rpm})$ until the removal of the organic solvents.

Particle Characterization. Particle morphology was studied using an Inspect F50 field emission gun scanning electron microscope. Samples were Au/Pd-coated (Leica EM ACE200, Leica, Wetzlar, Germany) to allow electrical observation. Particle size diameter was determined using ImageJ software (Version 1.48f, NIH, USA) $(N=150)$, and size distribution was analyzed by statistical analysis. The morphology and size distribution of the samples were also characterized by transmission electron microscopy (TEM, T20-FEI Tecnai thermoionic transmission electron microscope, Hillsboro, OR, USA). For TEM observation, samples were stained with phosphotungstic acid as negative contrast, following a previous protocol of our group. ${ }^{21}$ The hydrodynamic diameter and $Z$-potential value of the colloidal particles at neutral $\mathrm{pH}$ were determined by dynamic light scattering measurements using a Brookhaven 90 Plus (Holtsville, NY, USA) equipment. Each sample was measured three times.

Rif encapsulation efficiency (EE) and drug loading of the electrosprayed particles were determined by UV-vis spectrophotometry (Jasco V670, Jasco Applied Science, Eschborn, Germany) at a wavelength of $340 \mathrm{~nm}$ by dissolving $2 \mathrm{mg}$ of Rif-loaded particles in $1 \mathrm{~mL}$ of DMSO using three independent samples. At the wavelength of study, no polymer interference was observed. The EE and drug loading (DL) were calculated with eqs 1 and 2, respectively

$$
\begin{aligned}
& \mathrm{EE}(\%)=\frac{\text { mass of entrapped drug }(\mathrm{mg})}{\text { total mass of drug added }(\mathrm{mg})} \times 100 \\
& \mathrm{DL}(\%)=\frac{\text { mass of entrapped drug }(\mathrm{mg})}{\text { total mass of particles }(\mathrm{mg})} \times 100
\end{aligned}
$$

Rif EE and DL of nanoprecipitated particles were calculated by an indirect quantification. The collected nanoparticles were washed several times with milliQ water using an Amicon Ultra15 centrifugal filter unit with $30 \mathrm{kDa}$ cutoff. Supernatants were collected, and nonentrapped Rif was measured by UV-vis. EE (eq 1) and DL (eq 2) were calculated by considering the entrapped Rif as the difference between the total Rif added and the nonentrapped Rif in a mass balance.

Fourier-transform infrared (FTIR) spectra were recorded to evaluate if drug-polymer molecular interactions occur. FTIR spectra were obtained using a Bruker VERTEX 70 FTIR spectrometer (Bruker, Billerica, MA, USA) equipped with a Golden Gate diamond ATR accessory. Spectra were recorded by averaging 40 scans in the $4000-600 \mathrm{~cm}^{-1}$ wavenumber range at a resolution of $4 \mathrm{~cm}^{-1}$. Data evaluation was carried out using OPUS software from Bruker Optics.

An X-ray diffractometer (Empyrean, Malvern Panalytical, Malvern, UK) was used to record the X-ray diffraction (XRD) diffractograms of the different samples. The measuring conditions were as follows: $2 \theta$ angle ranging from 5 to 50 deg with a scan rate of $0.017^{\circ} / \mathrm{s}$ and a step size of $0.013^{\circ}$.

Particle stability under simulated gastric and intestinal conditions was analyzed using the paddle method under sink conditions. The corresponding particles were suspended in SGF without enzymes $\left(0.1 \mathrm{~N} \mathrm{HCl}, \mathrm{pH} 1.1\right.$ at $\left.37^{\circ} \mathrm{C}, 2 \mathrm{~h}\right)$. The particles were also immersed in intestinal simulated fluid (PBS, $\mathrm{pH} 7.4$ at $37{ }^{\circ} \mathrm{C}$ ) without enzymes, and their stability and morphology were analyzed by scanning electron microscopy (SEM) at different time points $(2 \mathrm{~h}, 24 \mathrm{~h}, 25$ days, and 60 days). Those long times were selected due to the mucoadhesive character of the microparticles, they do not dissolve at a specific $\mathrm{pH}$, they are $\mathrm{pH}$-independent, and they slowly erode releasing their contained cargo. 
EuRS 100
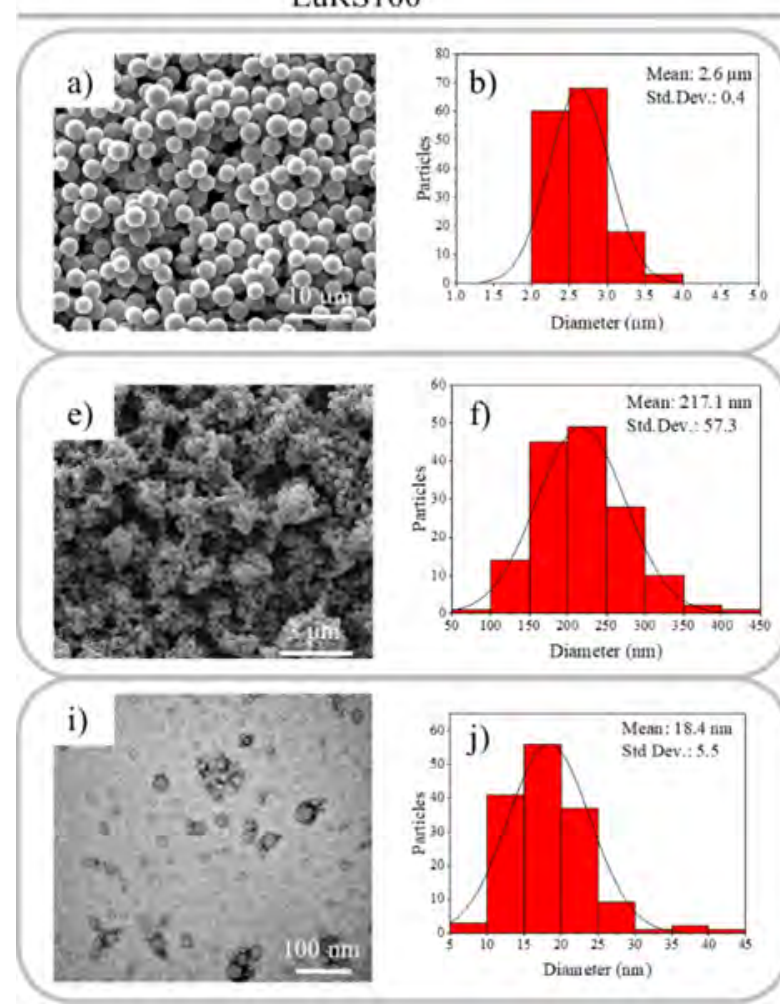

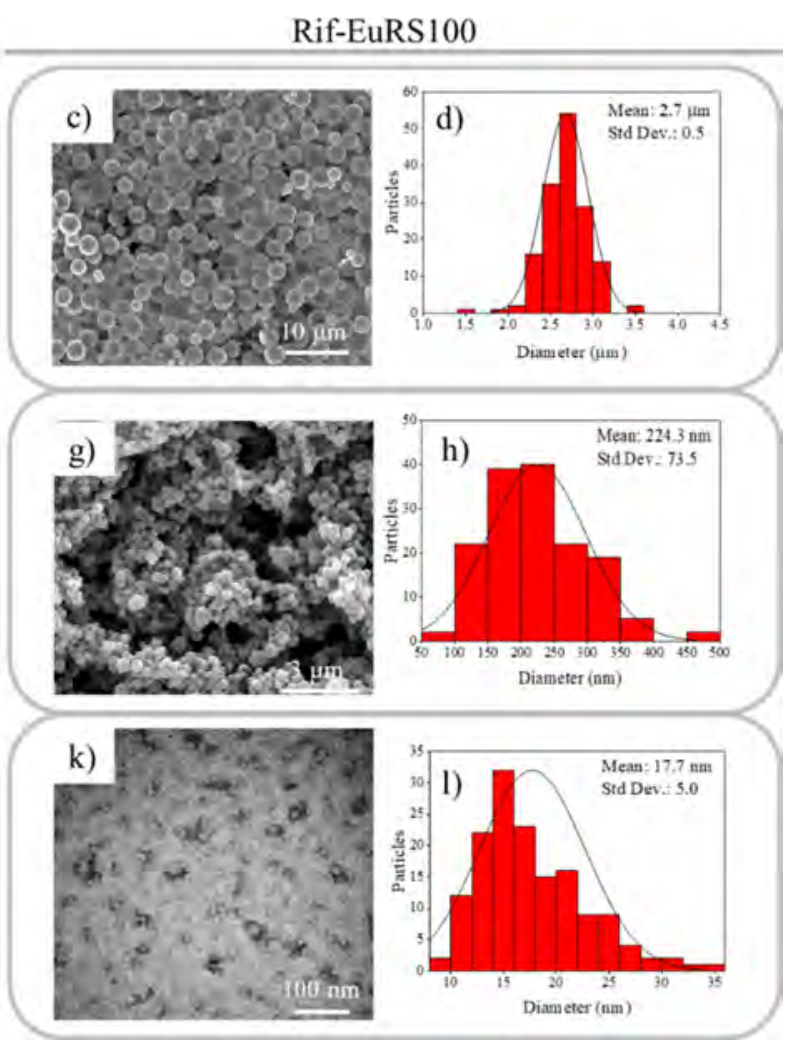

Figure 1. (a,b) SEM image and size distribution retrieved from SEM measurements of EuRS100 microparticles (electrospray), (c,d) SEM image and size distribution of Rif-loaded EuRS100 microparticles (electrospray), (e,f) SEM image and size distribution of empty EuRS100 submicroparticles (electrospray), (g,h) SEM image and size distribution of Rif-loaded EuRS100 sub-microparticles (electrospray), (i,j) TEM image and size distribution of empty EuRS100 nanoparticles (nanoprecipitation), and (k,l) TEM image and size distribution of Rif-loaded EuRS100 nanoparticles.

In vitro Drug Release Study. The in vitro release of Rif from the corresponding particles was performed under sink conditions by introducing $5 \mathrm{mg}$ of particles in $5 \mathrm{~mL}$ of SGF during $2 \mathrm{~h}$. Afterward, particles were centrifuged for $10 \mathrm{~min}$ at $13,300 \mathrm{rpm}$, and the supernatant was replaced with PBS $1 \times(5$ $\mathrm{mL}$ ) to simulate intestinal conditions. Particles were incubated at $37{ }^{\circ} \mathrm{C}$ under continuous mixing, and at predetermined time intervals $(0,0.5,1,2,4,6$, and $8 \mathrm{~h}$ and 1 to 75 days $), 1 \mathrm{~mL}$ of the supernatant was collected by centrifugation for $5 \mathrm{~min}$ at $13,300 \mathrm{rpm}$ and an equal volume of fresh PBS was replenished. To collect the supernatants of microfluidic-produced particles, an Amicon Ultra-15 centrifugal filter unit with $30 \mathrm{kDa}$ cutoff was used. Four different samples were independently analyzed. In PBS, the potential degradation of the drug at long times is minimized (see Figure S3).

Bacterial Culture and Viability Analysis. To determine the antimicrobial activity of the different particles, the minimum inhibitory concentration (MIC) and the MBC of the free antibiotic (Rif) were evaluated using S. aureus (ATCC 25923) as a model of a Gram-positive infective pathogen. Bacteria were grown overnight in TSB at $37{ }^{\circ} \mathrm{C}$ under continuous shaking, $150 \mathrm{rpm}$. When bacteria were grown at the stationary growth phase $\left(10^{10} \mathrm{cfu} / \mathrm{mL}\right)$, they were diluted in TSB until reaching $10^{5} \mathrm{cfu} / \mathrm{mL}$. The inoculum was placed into an eppendorf tube with a specific quantity of Rif (0-1 ppm) dissolved in DMSO. A toxicity control using only DMSO was also performed in parallel. After $24 \mathrm{~h}$, at $37^{\circ} \mathrm{C}$ and $150 \mathrm{rpm}$, the standard serial dilution method was used to determine viable bacteria. Positive control (untreated bacteria) was also included in the study and analyzed as reported.
The same protocol detailed to determine MIC and MBC values was here considered to evaluate the antimicrobial effect of the Rif-containing particles. A bacterial inoculum of $10^{5} \mathrm{cfu} /$ $\mathrm{mL}$ was placed with different particle dispersion concentrations $(1-0.005 \mathrm{mg} / \mathrm{mL})$, and the serial dilution method was again used. Each experiment was performed in triplicate with four replicas for each sample using independent syntheses.

Cell Culture and Viability Analysis. Caco2-TC7 human epithelial colorectal adenocarcinoma cells and human dermal fibroblast were used to evaluate the potential cytotoxic effect of EuRS100 and Rif-EuRS100 particles. Caco 2 and fibroblast cells were cultured in high-glucose DMEM supplemented with $10 \%$ (v/v) FBS and $1 \%(\mathrm{w} / \mathrm{v})$ antibiotic-antimycotic at $37^{\circ} \mathrm{C}$ in a humidified atmosphere with $5 \% \quad \mathrm{CO}_{2}$. Cytotoxicity was measured analyzing the cell metabolism using the Blue cell viability assay according to the manufacturer's instructions. Briefly, cells were seeded into 96-well plates, allowed to attach and proliferate until confluence, and treated with empty particles, Rif-containing particles, and comparative doses of the free antibiotic. Particles were tested at concentrations between 1 and $0.005 \mathrm{mg} / \mathrm{mL}$, and the free antibiotic at the measured value of Rif concentration released after $24 \mathrm{~h}$ from each particle was also evaluated for $24 \mathrm{~h}$ at $37^{\circ} \mathrm{C}$ and $5 \% \mathrm{CO}_{2}$. After incubation, cells were washed several times to remove particles from the wells. Then, cells were incubated with the reagent $\left[10 \%(\mathrm{v} / \mathrm{v})\right.$ in DMEM] and incubated for $3 \mathrm{~h}$ at $37{ }^{\circ} \mathrm{C}$. Fluorescence was measured at $530 / 590 \mathrm{~nm}$ excitation/ emission wavelengths in a Multimode Synergy HT microplate reader (Biotek, WI, USA). Cell viability was determined by comparing the values retrieved for the treated cells and the 
Table 1. Encapsulation Efficiency (\%), Drug Loading (\%), and Z-Potential (mV) of Electrosprayed Rif-Loaded Microparticles, Electrosprayed Rif-Loaded Sub-Microparticles, and Rif-Loaded Nanoparticles Obtained by Microfluidic Nanoprecipitation

\begin{tabular}{|c|c|c|c|c|}
\hline & EE (\%) & drug Loading (mg rif/mg particle) & drug loading (\%) & $Z$-potential $(\mathrm{mV})$ \\
\hline EuRS100 13\%-ES & $99.3 \pm 1.6$ & $0.13 \pm 0.003$ & $13.0 \pm 0.3$ & $+28.9 \pm 2.6$ \\
\hline EuRS100 3\%-ES & $82.9 \pm 1.0$ & $0.14 \pm 0.002$ & $14.0 \pm 0.2$ & $+23.9 \pm 2.7$ \\
\hline EuRS100 5\%-NP & $42.3 \pm 2.4$ & $0.02 \pm 0.001$ & $2.0 \pm 0.1$ & $+17.4 \pm 2.2$ \\
\hline
\end{tabular}

a)
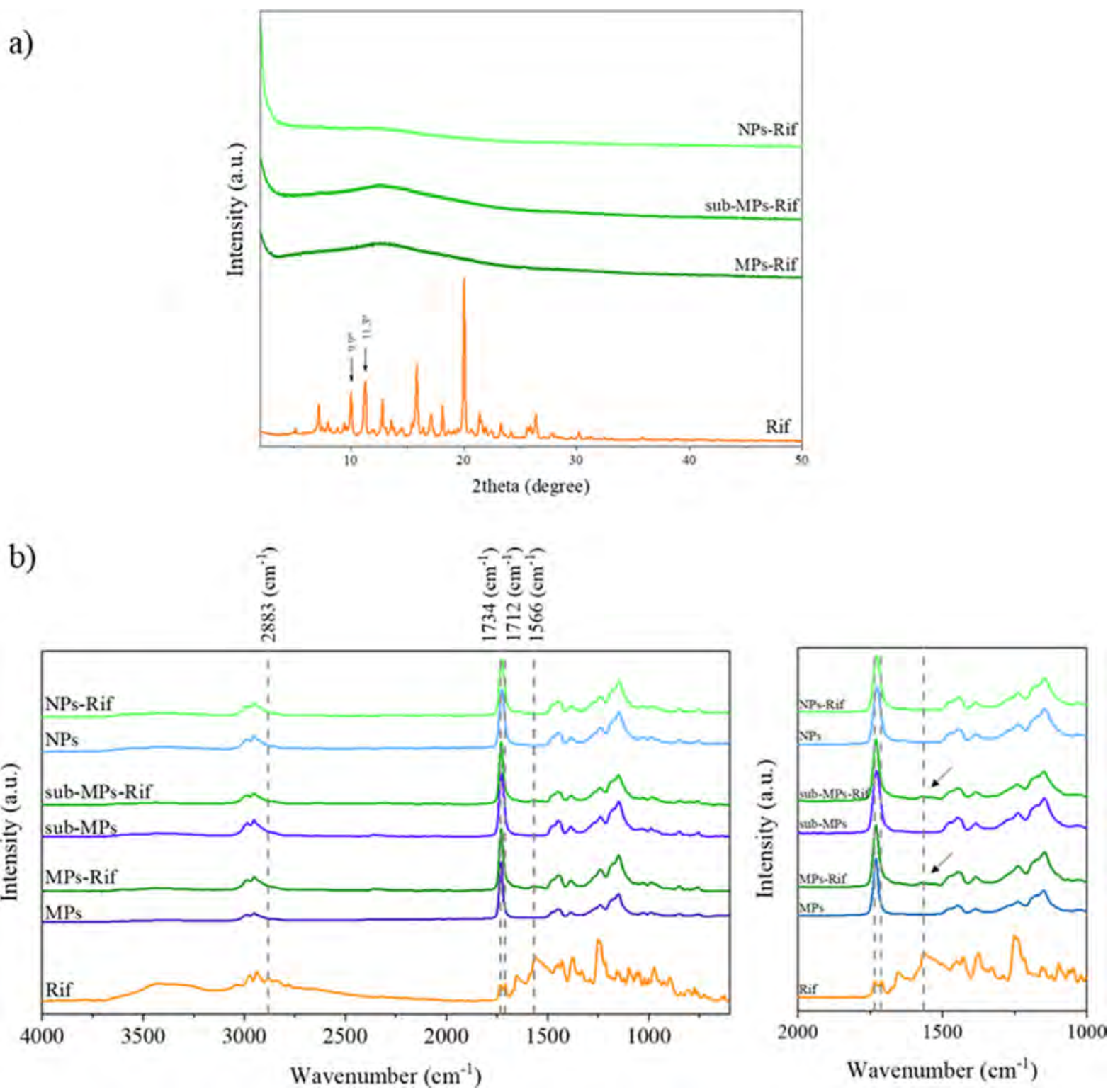

Figure 2. a) XRD diffractograms and (b) FTIR spectra of unloaded EuRS100 microparticles, sub-microparticles, and nanoparticles; Rif-loaded microparticles, sub-microparticles, and nanoparticles, and free Rif.

untreated controls which were assigned with $100 \%$ viability. Each determination was performed in triplicate having four replicas in each experiment, using independent syntheses.

The subcytotoxic dose was reached when the viability of cell lines was reduced more than $30 \%$ (subcytotoxic concentration). At this dose, the effect of the particles on the cell membrane was determined by the study of cell apoptosis by flow cytometry. After treating the cells with the particles, cells were exhaustively washed and stained with annexin V-FITC and propidium iodide. Finally, stained cells were incubated for 15 min and then analyzed in a Facsaria BD equipment. Data were evaluated using Facsdiva BD software. Control samples with untreated cells were also analyzed to determine the influence of the particles on cell apoptosis. The cell cycle at subcytotoxic concentrations was also analyzed. After treatment, cells were collected in PBS and fixed in 70\% cold ethanol and stored at $4{ }^{\circ} \mathrm{C}$ for $24 \mathrm{~h}$. Then, samples were treated with RNase A, and DNA was stained with propidium iodide. Samples were measured in a Facsarray BD equipment with Modifit 3.0 Verity software. Control samples having untreated cells were also analyzed to determine the influence of the particles on the cell cycle. 
a)

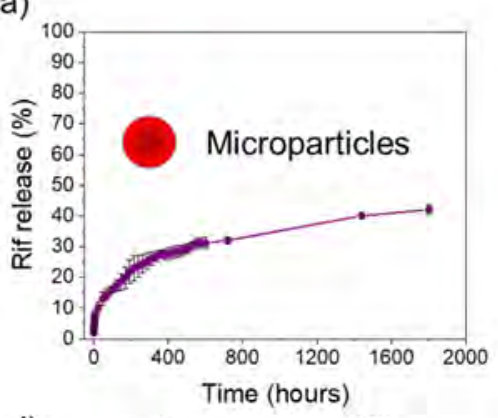

d)

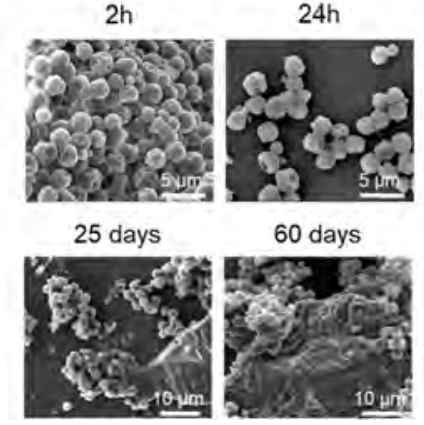

b)

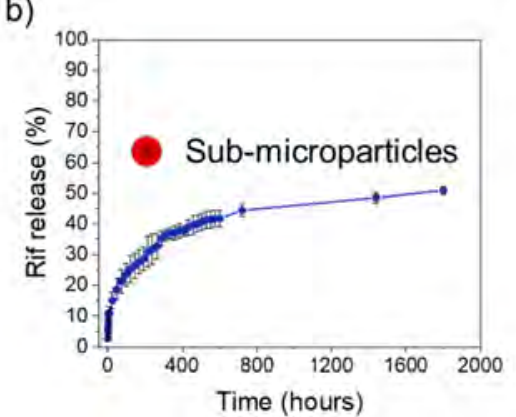

e)
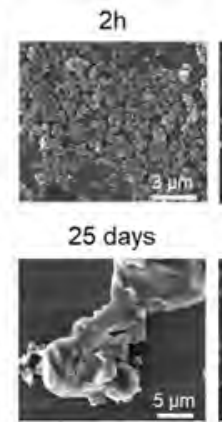

c)

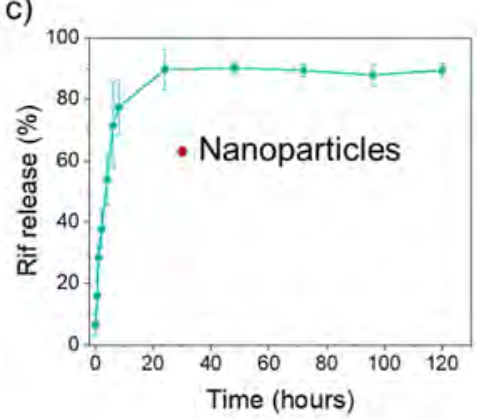

f)

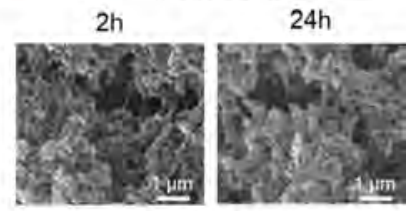

7 days

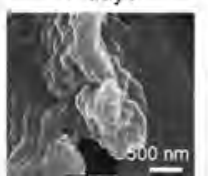

Figure 3. Rif release from (a) electrosprayed Rif-loaded EuRS100 microparticles, (b) electrosprayed Rif-loaded EuRS100 sub-microparticles, and (c) nanoprecipitated Rif-loaded EuRS100 nanoparticles. SEM images of Rif-loaded EuRS100 carriers at different release times under SGF (the first $2 \mathrm{~h}$ ) and SIF (afterward): (d) electrosprayed Rif-loaded EuRS100 microparticles, (e) electrosprayed Rif-loaded EuRS100 sub-microparticles, and (f) nanoprecipitated Rif-loaded EuRS100 nanoparticles.

Statistical Analysis. All results are expressed as mean \pm standard deviation (SD). Two-way analysis of variance (ANOVA) was used to analyze bacterial and cellular experiments (GraphPad Prism 7, San Diego, USA). Statistically significant differences were considered when $p \leq 0.05$.

\section{RESULTS}

Physicochemical Characterization of the Particles. Using two different fabrication techniques (i.e., electrospraying and nanoprecipitation) and varying the solvents (acetone, ethanol, and chloroform) and polymer concentrations, three different particle sizes in the nano-, submicro-, and microscale were able to be produced using EuRS100. An initial battery of solvents, polymer concentrations used in the polymeric precursor solution, and Rif concentrations was screened in order to optimize the corresponding syntheses and obtain high DL and reduced particle polydispersity (optimization results not shown). Figure $1 \mathrm{a}, \mathrm{b}$ shows the SEM image and size distribution of empty microparticles made with EuRS100 13\% $(\mathrm{w} / \mathrm{v})$ in chloroform obtained by electrospraying, having a uniform particle size distribution centered at $2.6 \pm 0.4 \mu \mathrm{m}$. When Rif was incorporated $(15 \% \mathrm{w} / \mathrm{w})$ in the same formulation, similar sizes $(2.7 \pm 0.5 \mu \mathrm{m})$ and particle monodispersity were also maintained (Figure $1 \mathrm{c}, \mathrm{d}$ ). To reduce the particle sizes of EuRS100 formulations, we considered to (1) use a mixture of ethanol and acetone instead of using chloroform in order to increase solvent polarity and (2) reduce the EuRS100 polymer concentration in the precursor solution. Figure 1e,f shows the SEM image and size distribution of empty microparticles made with EuRS100 3\% (w/v) in EtOH/ acetone obtained by electrospraying, also having a uniform submicron particle size distribution centered at $271.1 \pm 57.3$ $\mathrm{nm}$. When Rif was incorporated $(20 \% \mathrm{w} / \mathrm{w})$ in the same formulation, similar sizes $(224.3 \pm 73.5 \mathrm{~nm})$ and particle monodispersity were also obtained (Figure 1g,h). Finally, in order to obtain particles in the nanoscale range, nanoprecipitation using miscible solvents in a commercial interdigital micromixer was used. Figure $1 \mathrm{i}, \mathrm{j}$ shows the SEM image and size distribution of empty nanoparticles made with EuRS100 5\% (w/v) in EtOH/acetone obtained by microfluidic-assisted nanoprecipitation, having a uniform particle size distribution centered at $18.4 \pm 5.5 \mathrm{~nm}$. When Rif was incorporated $(5 \% \mathrm{w} / \mathrm{w})$ into the same formulation, similar sizes $(17.7 \pm 5.0 \mathrm{~nm})$ and particle monodispersity were also obtained (Figure $1 \mathrm{k}, \mathrm{l}$ ).

In electro-hydrodynamic spraying, flash solvent evaporation takes place during the flight between the needle tip and the collector caused by the high volatility of the organic solvents used, whereas the polymer precipitates trapping the associated drug. However, during nanoprecipitation, because of the miscibility of the solvent and antisolvent, part of the entrapped drug diffuses with the organic solvent during solvent displacement, and at the same time, the polymer starts to precipitate, trapping the remaining drug because of its immiscibility in the antisolvent phase. For this reason, Rif loadings (Table 1$)$ obtained for the micro- $(13 \pm 0.3 \%)$ and sub-microparticles $(14 \pm 0.2 \%)$ by electrospraying were higher than the ones measured for the nanoparticles $(2 \pm 0.1 \%)$ obtained in the microfluidic mixer. These results are according to those reported in the previous literature where higher $\mathrm{EE}$ was obtained when particle sizes increased. ${ }^{22}$ Likewise, drug encapsulation efficiencies for the particles obtained by electrospraying were at least double than the ones obtained when using the nanoprecipitation method $(42.3 \pm 2.4 \%)$. In the production of the electrosprayed particles when using a reduced polymer concentration (RS100 3\%, w/v), the EE $(82.9 \pm 1 \%)$ was lower than the one obtained when using high polymer concentration RS100 13\% (w/v) $(99.3 \pm 1.6 \%)$, probably because of a reduced mixture viscosity and because of drug evaporation during the electrohydrodynamic synthesis 
process. The zeta potential for the colloidal suspensions of the obtained particles in water was largely positive because of the presence of $5 \%$ of functional quaternary ammonium groups in the EuRS100, which confers mucoadhesive properties. ${ }^{23}$ This positive charge facilitates particle interaction with the negatively charged acidic amino acids (i.e., aspartic acid and glutamic acid) and acidic monosaccharides (i.e., sialic acid) present in the intestinal mucus. ${ }^{24}$

XRD diffractograms and FTIR spectra of Rif and the different particles are shown in Figure 2. Peaks at 9.9 and $11.3^{\circ}$ in Rif XRD diffractogram (Figure 2a) indicate that the used drug was in the polymorphic form II. This was confirmed by FTIR analysis, where the Rif spectrum shows bands at 1734, 1712 , and $1567 \mathrm{~cm}^{-1}$ (Figure $2 \mathrm{~b}$ ) related to furanone $\mathrm{C}=\mathrm{O}$, acetyl $\mathrm{C}=\mathrm{O}$, and amide $\mathrm{C}=\mathrm{O}$, respectively, characteristic of form II Rif. ${ }^{25}$ The small band at $2883 \mathrm{~cm}^{-1}$ related to $\mathrm{N}-\mathrm{CH}_{3}$ vibration can also be observed. Loaded particle XRD diffractograms do not show any peak that could be related to a crystalline form of Rif. Even when this fact could be due to the presence of amorphous drug inside the particles, the absence of signals could also be related to the presence of drug crystals smaller than $40 \AA$ and/or because the Rif load is below the technique detection limit. The broad band observed in particle diffractograms confirms the amorphous nature of the polymer. ${ }^{26,27}$ Loaded and unloaded particle FTIR spectra have a characteristic peak at $1726 \mathrm{~cm}^{-1}$, assigned to the EuRS100 $\mathrm{C}=\mathrm{O}$ ester vibration. ${ }^{28}$ No peak related to Rif can be observed in loaded nanoparticle spectra, while a small band observed around $1566 \mathrm{~cm}^{-1}$ would be related to the presence of Rif in micro- and sub-microparticles. The amide $\mathrm{C}=\mathrm{O}$ vibration band appears at almost the same wavelength for Rif forms I and II (1567 and $1566 \mathrm{~cm}^{-1}$, respectively), and as a consequence, it is impossible to know, from this peak, the polymorphic form of Rif in the particles or even their crystallinity. The absence of Rif signals in the FTIR nanoparticle spectrum could be due to the low drug loading achieved by the nanoprecipitation technique.

Drug Release Kinetics and Morphological Analysis under Simulated Enteric Conditions. Cumulative drug release kinetics placing the particles under simulated gastric conditions for $2 \mathrm{~h}$ followed by intestinal simulated fluid conditions (up to 60 days) are depicted in Figure 3. After $2 \mathrm{~h}$ of contact in SGF, only $(2.1 \pm 0.2 \%),(2.8 \pm 0.6 \%)$, and $(6.5 \pm$ $3.5 \%)$ of the drug were released from the Rif-loaded EuRS100 microparticles $(\sim 2 \mu \mathrm{m})$, EuRS100 sub-microparticles $(\sim 200$ $\mathrm{nm})$, and EuRS100 nanoparticles $(\sim 20 \mathrm{~nm})$, respectively. A homogenous particle surface free of cracks or pinholes might be responsible for this protection under SGF (Figure S1). The smaller the particle size, the faster the Rif release in both SGF and simulated intestinal fluid (SIF). In this regard, Rif-loaded EuRS100 nanoparticles $(\sim 20 \mathrm{~nm})$ showed a burst release with almost $89 \%$ released after 24 h. EuRS100 sub-microparticles $(\sim 200 \mathrm{~nm})$ released almost $51 \%$ of their contained drug in 75 days, whereas the EuRS100 microparticles $(\sim 2 \mu \mathrm{m})$ released $42 \%$ of the encapsulated drug in the same time period. For microparticles, different from nanoparticles, the small surface area per unit volume leads to a reduced rate of water permeation. Specifically, the surface to volume ratio in $20 \mathrm{~nm}$ nanoparticles $\left(0.3 \mathrm{~nm}^{-1}\right)$ is 100 -fold larger than that in $2 \mu \mathrm{m}$ microparticles $\left(0.003 \mathrm{~nm}^{-1}\right)$. Then, the limited diffusivity in the polymeric matrix reduces the maximum possible rate of encapsulated Rif release. As a result, Rif is released in a timecontrolled manner from the $\mathrm{pH}$-independent low-permeability mucoadhesive particles, and their different sizes allow reaching pharmacokinetic control. Therefore, having the same polymer, by just modifying the particle size, drug release kinetics can be tuned depending on the needs. The EuRS100 nanoparticles $(\sim 20 \mathrm{~nm})$ would provide with a fast antibiotic release, and the larger EuRS100 submicron $(\sim 200 \mathrm{~nm})$ or microparticles $(\sim 2$ $\mu \mathrm{m}$ ) would provide time-dependent sustained release.

Drug release data of all the particles synthesized were fitted using different mathematical models including KorsmeyerPeppas, Higuchi, Peppas-Sahlin, Lindner-Lippold, zero-order, and first-order kinetics (Figure S2).

The best fit for the EuRS100 submicron particles ( 200 $\mathrm{nm})$ and microparticles $(\sim 2 \mu \mathrm{m})$ synthesized by electrospraying was observed for the Peppas-Sahlin model (Table S1), ${ }^{29}$ where $K_{1}$ is the kinetic constant related to the diffusional contribution, $K_{2}$ is the kinetic constant related to the case-II polymer relaxation contribution, and the coefficient $\mathrm{n}$ is related to the diffusion type. In this case, diffusional mechanism predominates over the relaxation of polymer chains $\left(K_{1} \gg K_{2}\right)$. The negative values obtained for $K_{2}$ would be related to the insignificant contribution of relaxation mechanisms compared to the diffusion process. ${ }^{30}$ In both cases, $n$ was lower than 0.43 , the expected value for Fickian diffusion from spherical geometries. Similar results were previously attributed to the effect of different particle size distributions. ${ }^{31}$ The best fit for the particles obtained with the microfluidic nanoprecipitated EuRS100 nanoparticles $(\sim 20 \mathrm{~nm})$ was observed for the Lindner-Lippold model (Table $\mathrm{S} 1)^{32}$ where $k$ is a release constant related to the release rate, $\mathrm{n}$ is the coefficient related to the release mechanism, and $b$ is the constant characteristic of the burst effect. When drug diffusion and polymer erosion control the release process in equal parts, the value of $n$ in the Lindner-Lippold model takes a value of 0.62 , and higher values of $n$ are attributed to an increase in erosion-driven control. In our case, $n=0.7$ would imply a slight predominance of erosion on the release process.

Figure $3 \mathrm{~d}-\mathrm{f}$ shows the morphology of the corresponding Rif-loaded EuRS100 particles after simulated gastric and intestinal conditions. SEM micrographs demonstrate their morphological stability under simulated gastric conditions $(0.1$ $\mathrm{N} \mathrm{HCl}, \mathrm{pH} 1.1$ at $37{ }^{\circ} \mathrm{C}, 2 \mathrm{~h}$ ), corroborating their ability to withstand an acidic environment. Under simulated intestinal conditions (PBS, pH 7.4 at $37^{\circ} \mathrm{C}$ for $24 \mathrm{~h}$ ), the particles slowly swelled and eroded, increasing their permeability. Different behaviors were observed for the particles after 25 and 60 days under these conditions, depending on the formulation. Despite the nonbiodegradable nature of these polycationic acrylic polymers, the EuRS100 when formulated as nanoparticles $(\sim 20 \mathrm{~nm})$ after being immersed for 7 days under simulated intestinal conditions formed plasticized agglomerations. Submicron particles $(\sim 200 \mathrm{~nm})$ initially swelled and eroded, and after 25 and 60 days under these conditions, they agglomerated and formed heterogeneous plasticized films. At the same time points, EuRS100 microparticles $(\sim 2 \mu \mathrm{m})$ still preserved most of their initial morphology, although incipient agglomeration and irreversible aggregation took place.

Even when some erosion could be observed in microparticles and sub-microparticles, this effect is not strong enough to lead drug release, and for these particles, a diffusioncontrolled process was observed. On the other hand, nanoparticles are more affected by the neutral medium because of their high surface per volume ratio, causing the observed burst release and rapid release kinetics. 
a) Theoretical free rifampicin ( $\mu \mathrm{g} / \mathrm{mL})$

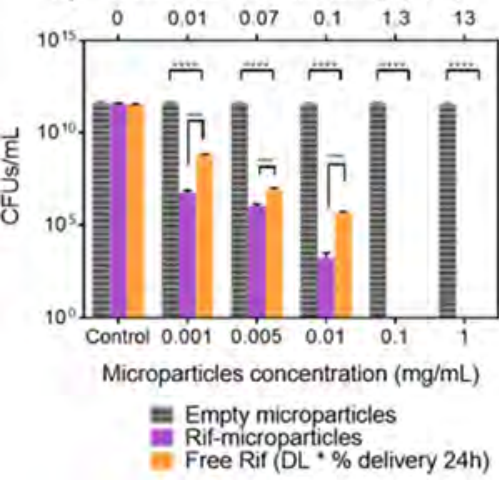

b) Theoretical free rifampicin $(\mu \mathrm{g} / \mathrm{mL})$

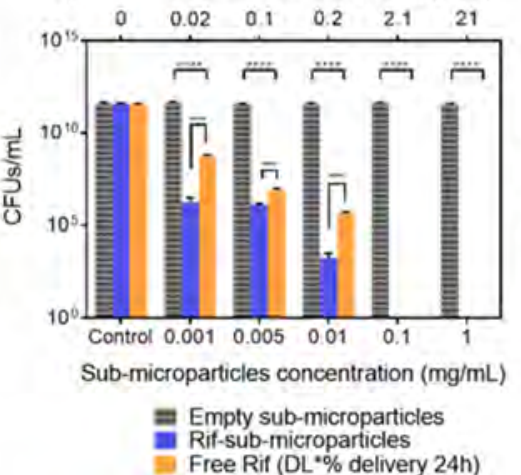

C) Theoretical free rifampicin ( $\mu \mathrm{g} / \mathrm{mL})$

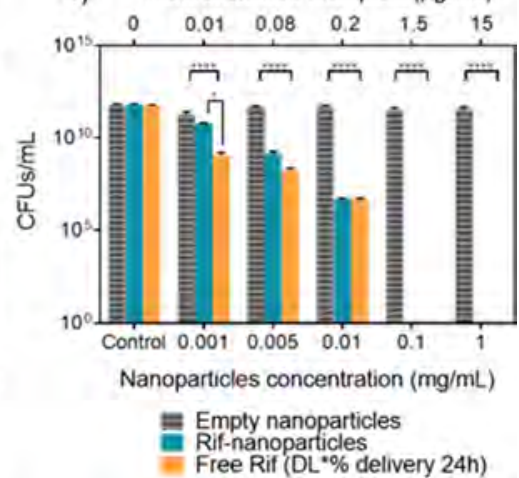

Figure 4. Antibacterial activity of (a) electrosprayed Rif-loaded EuRS100 microparticles, (b) electrosprayed Rif-loaded EuRS100 submicroparticles, and (c) Rif-loaded EuRS100 nanoprecipitated nanoparticles. The control sample was assigned to the untreated bacteria. Data are presented as mean \pm SD $(n=3) .(* p<0.05, * * p<0.01, * * * p<0.001$, and $* * * * p<0.0001)$.

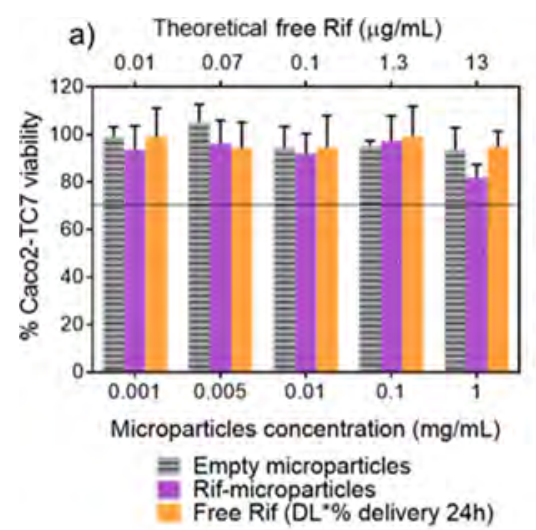

d) Theoretical free Rif ( $\mu \mathrm{g} / \mathrm{mL})$

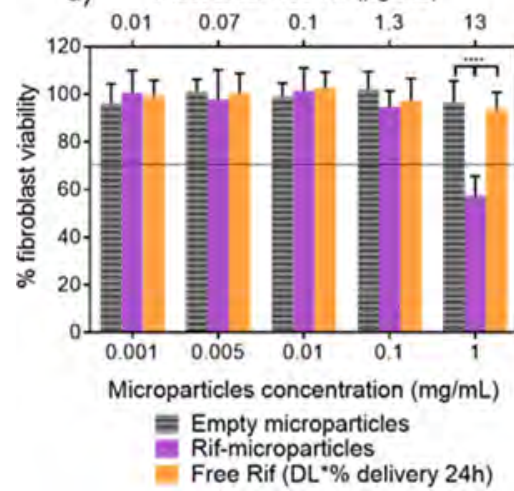

b) Theoretical free Rif ( $\mu \mathrm{g} / \mathrm{mL})$

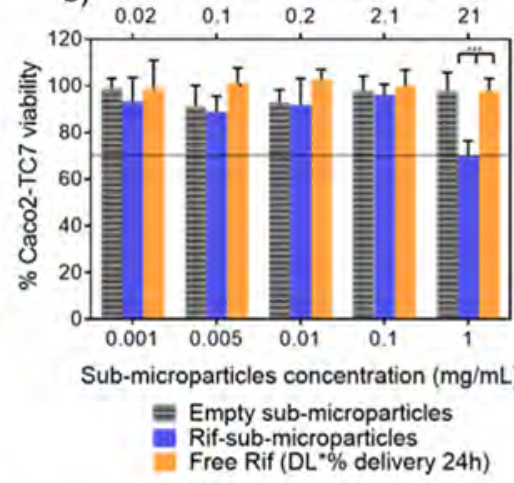

e) Theoretical free Rif $(\mu \mathrm{g} / \mathrm{mL})$

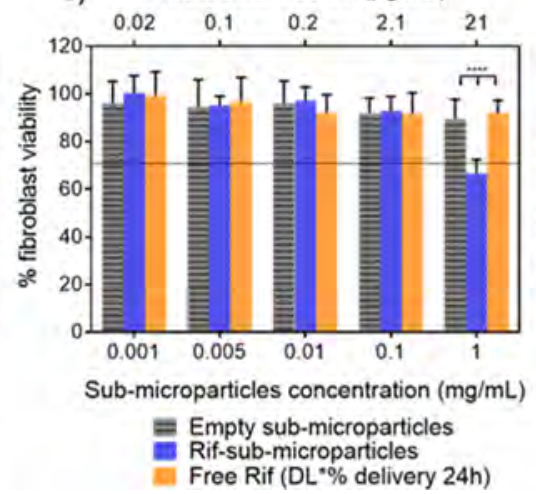

c) Theoretical free Rif $(\mu \mathrm{g} / \mathrm{mL})$

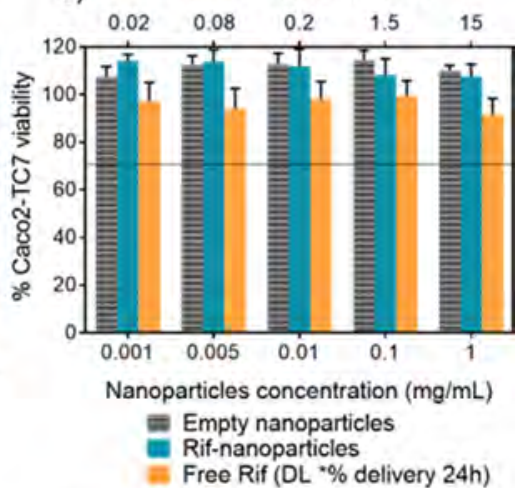

f) Theoretical free Rif $(\mu \mathrm{g} / \mathrm{mL})$

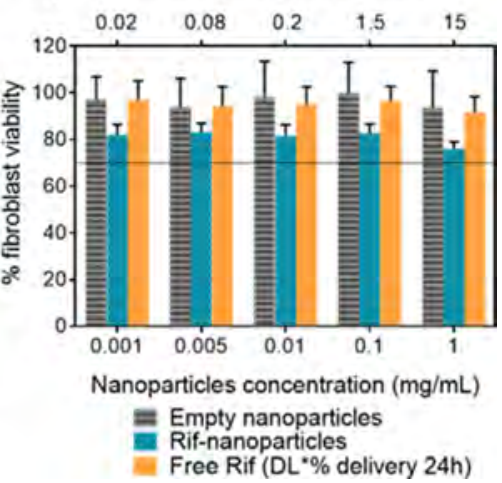

Figure 5. (a-c) Cell viability in the Caco2-TC7 cell line of (a) electrosprayed Rif-loaded EuRS100 microparticles, (b) electrosprayed Rif-loaded EuRS100 sub-microparticles, and (c) nanoprecipitated Rif-loaded EuRS100 nanoparticles. (d-f) Cell viability in the fibroblast cell line of (d) electrosprayed Rif-loaded EuRS100 microparticles, (e) electrosprayed Rif-loaded EuRS100 sub-microparticles, and (f) nanoprecipitated Rif-loaded EuRS100 nanoparticles. $100 \%$ viability was assigned to the untreated control cells. Data are presented as mean \pm SD $(n=3)$. $(* p<0.05, * * p<$ $0.01, * * * p<0.001$, and $* * * * p<0.0001)$.

Antimicrobial Activity and Cytocompatibility. These materials were challenged against $S$. aureus ATCC 25923 to corroborate that the activity of Rif was still preserved after encapsulation. MIC and MBC values for free Rif were calculated using the standard broth microdilution method. It can be observed that the MIC was reached at $0.05-0.125 \mu \mathrm{g} /$ $\mathrm{mL}$, whereas the MBC was reached for concentrations in the $0.25-0.5 \mu \mathrm{g} / \mathrm{mL}$ range, in agreement with the previous literature. $^{33,34}$ Comparing the antibacterial effect in the inhibition of ribonucleic acid synthesis of the free drug and the equivalent doses of the EuRS100-encapsulated Rif, the particles and the free drug showed the same antibacterial activity at high doses $(>0.1 \mathrm{mg} / \mathrm{mL}$ for the particles which correspond to a free antibiotic released concentration of $>1.3$ $\mu \mathrm{g} / \mathrm{mL}$ in $24 \mathrm{~h}$ ) independent of their sizes (Figure 4). However, at low concentrations (between 0.001 and $0.01 \mathrm{mg}$ / $\mathrm{mL}$ for the particles, which correspond to a free antibiotic released concentration in $24 \mathrm{~h}$ between 0.01 and $0.1 \mu \mathrm{g} / \mathrm{mL}$ ), a superior antimicrobial effect was observed for the encapsulated micro- $(\sim 2 \mu \mathrm{m})$ and sub-microparticles $(\sim 200 \mathrm{~nm})$, probably promoted by a contact effect by the electrostatic interaction between the particle (positive superficial charge) and the 
a)

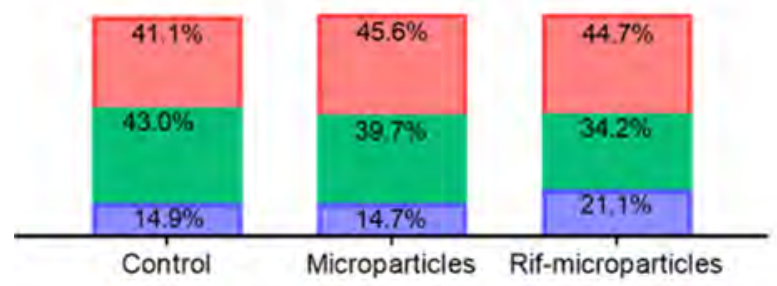

b)

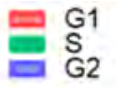

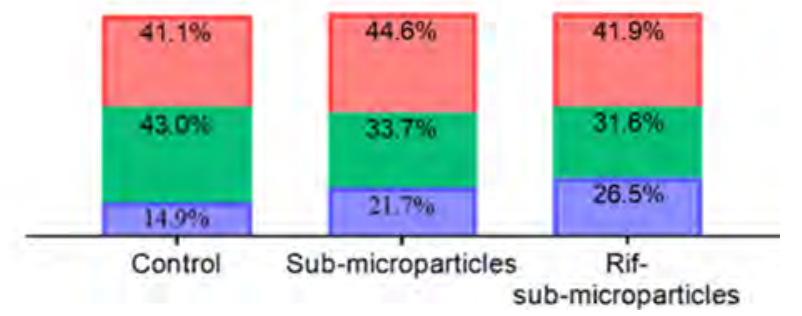

Figure 6. Cell cycle assay in the fibroblast cell line of (a) electrosprayed EuRS100 microparticles and (b) electrosprayed EuRS100 submicroparticles. G1 (first growth phase), S (DNA replication phase), and G2 (growth phase). Control samples (not treated cells) were analyzed as the background cell cycle level.

Table 2. Cell Apoptosis Evaluation by Flow Cytometry after Treatment with Rif-Loaded Microparticles and SubMicroparticles on the Fibroblast Cell Line ${ }^{a}$

$\begin{array}{lccccc} & \begin{array}{c}\text { control } \\ (\%)\end{array} & \begin{array}{c}\text { EuRS100 microparticles } \\ (\%)\end{array} & \begin{array}{c}\text { Rif-EuRS100 microparticles } \\ (\%)\end{array} & \begin{array}{c}\text { EuRS100 sub-microparticles } \\ (\%)\end{array} & \begin{array}{c}\text { Rif-EuRS100 sub-microparticles } \\ (\%)\end{array} \\ \text { viability } & 76.9 & 81.7 & 76.6 & 78.5 \% & 78.5 \\ \text { early apoptosis } & 4.3 & 3.6 & 8.5 & 4.1 & 4.4 \\ \text { late apoptosis } & 14.9 & 9.57 & 13.3 & 13.5 & 7.1 \\ \text { necrosis } & 3.9 & 5.05 & 1.5 & 3.8 & 9.9\end{array}$

${ }^{a}$ Control samples (not treated cells) were analyzed as the background apoptosis level.

bacteria (negatively charged cell wall). For the EuRS100, when formulated as nanoparticles $(\sim 20 \mathrm{~nm})$, a similar antimicrobial effect was observed for the free and for the encapsulated antibiotics in these low-range concentrations. The parent EuRS100 particles nonloaded with the antibiotic did not display any antimicrobial action at the doses tested. Therefore, the results shown in Figure 4 would infer that particle doses as low as $0.1 \mathrm{mg} / \mathrm{mL}$ for the microparticles $(\sim 2 \mu \mathrm{m})$, the submicroparticles $(\sim 200 \mathrm{~nm})$, and the nanoparticles $(\sim 20 \mathrm{~nm})$ would be needed to reach MBC.

The same doses of the empty and Rif-loaded EuRS100 particles were also evaluated using human cell lines (Caco-2, Human Caucasian colon adenocarcinoma cell line and human dermal fibroblasts) in order to corroborate the reduced toxicity of the materials at the doses that produced a complete bacterial eradication. It can be observed (Figure $5 a-c$ ) that no cytotoxic effect was observed for any of the particles tested at concentrations below $1 \mathrm{mg} / \mathrm{mL}$ for Caco-2 cells having viability percentages higher than $70 \%$, but it is important to highlight that at these concentrations, a bactericidal effect was observed (Figure 4). However, the treatment of fibroblasts, assayed (Figure $5 \mathrm{~d}-\mathrm{f}$ ) with micro- and sub-microparticles at a concentration of $1 \mathrm{mg} / \mathrm{mL}$, affected cell viability with percentages of $57.4 \%$ for microparticles and $66.7 \%$ for submicroparticles, but no effect was observed for the nanoparticles at the doses tested (Figure 5c).

Following the recommendations of the ISO 10993-5, in which viability percentages below $70 \%$ are considered cytotoxic, we selected a concentration of $1 \mathrm{mg} / \mathrm{mL}$ to corroborate the non-cytotoxicity of the submicron particles and microparticles, evaluating the effect of the Rif-loaded particles on the cell cycle arrest and potential apoptosis induction. As it can be seen in Figure 6, no significant differences compared to the untreated controls were observed for the particle-treated cells independently if the particles were loaded with the Rif or not. Compared to the controls, an increase in the G2 phase (i.e., rapid cell growth and synthesis of proteins preceding mitosis) of the cell cycle was observed for the Rif-loaded particles. The results showed that no DNA damage or cell cycle arrest was observed for Rif-loaded microparticles (Figure $6 \mathrm{a}$ ) and sub-microparticles (Figure 6b) in the fibroblasts.

By comparing the apoptosis induction of the empty and Rifloaded particles $(1 \mathrm{mg} / \mathrm{mL})$ on the fibroblasts with the nontreated cells, the effect of the particles on the cell membrane was evaluated (Table 2). Necrosis showed an increase lower than $6 \%$, while apoptosis showed a maximum increase of $2.65 \%$ for the Rif-loaded microparticles. Nevertheless, apoptosis percentage displayed a decrease of up to $7.73 \%$ for the rest of the particles tested. Rif-loaded and unloaded submicron particles and microparticles did not exert cytotoxic effects; hence, the reduction on cell viability for a concentration of $1 \mathrm{mg} / \mathrm{mL}$ was probably caused by experimental manipulation. Previous studies have shown the absence of cytotoxic effects of EuRS100 particles on SW480 cell lines (colon adenocarcinoma cell line) ${ }^{35}$ and on HCEpC cell lines (human corneal epithelial cells) at a concentration of $100 \mu \mathrm{g} / \mathrm{mL} .^{36}$ Cell viabilities over $80 \%$ for Caco- 2 cell lines at a concentration of up to $20 \mathrm{mg} / \mathrm{mL}$ of EuRS100 microparticles were also reported. ${ }^{37}$ In other studies, in vitro Rif treatment was not harmful on HepG2 cell lines (liver hepatocellular carcinoma), ${ }^{38}$ neither on primary murine macrophages. ${ }^{39}$ However, it has been reported that at high doses reached by systemic administration, Rif may be detrimental to cell proliferation of osteogenic cells. ${ }^{40}$

Pharmacokinetic Control on Drug Release. Finally, we wanted to demonstrate that using the different Rif-loaded particles developed, it was possible to reach MIC or MBC at different time points depending on the needs. We used the required amounts of EuRS100 nanoparticles $(\sim 20 \mathrm{~nm})$, submicron particles $(\sim 200 \mathrm{~nm})$, and microparticles $(\sim 2 \mu \mathrm{m})$ that contain the same amount of Rif $(0.05 \mathrm{mg})$ in all cases. 
a)

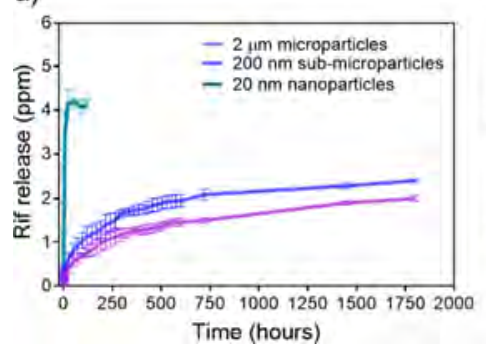

b)

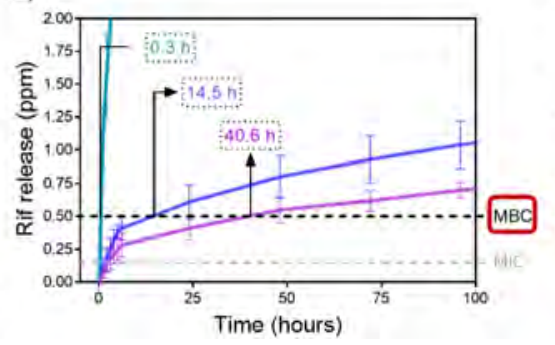

c)

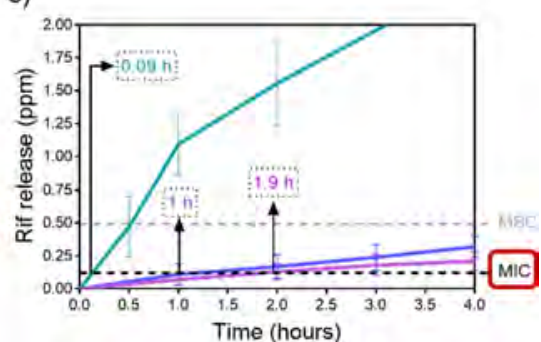

Figure 7. (a) Evolution of the relation observed between time and Rif release in the Rif-loaded EuRS100 carriers developed in this work, (b) time to reach $\mathrm{MBC}$, and (c) time to reach $\mathrm{MIC}$.

With this aim, $2.5 \mathrm{mg}$ of EuRS100 nanoparticles $(\sim 20 \mathrm{~nm})$, $0.36 \mathrm{mg}$ of EuRS100 submicron particles $(\sim 200 \mathrm{~nm})$, and 0.39 mg of EuRS100 microparticles $(\sim 2 \mu \mathrm{m})$ were placed in $10 \mathrm{~mL}$ of PBS simulating intestinal fluid at $37{ }^{\circ} \mathrm{C}$. Figure $7 \mathrm{~b}$ shows that using the EuRS100 when formulated as nanoparticles $(\sim 20 \mathrm{~nm})$, the MBC can be reached in only $0.3 \mathrm{~h}$, whereas with the submicron EuRS100 particles $(\sim 200 \mathrm{~nm})$, the same MBC concentration is reached in $14.5 \mathrm{~h}$, and with the EuRS100 microparticles $(\sim 2 \mu \mathrm{m})$, this concentration can be reached after $40.6 \mathrm{~h}$. Figure $7 \mathrm{c}$ also shows that concentrations above the MIC can be reached in only $0.09 \mathrm{~h}$ when using small EuRS100 nanoparticles $(\sim 20 \mathrm{~nm})$, whereas with the submicron EuRS100 particles $(\sim 200 \mathrm{~nm})$, it would be reached after $1 \mathrm{~h}$, and with the EuRS100 microparticles $(\sim 2 \mu \mathrm{m})$, it would be reached after $1.9 \mathrm{~h}$.

\section{DISCUSSION}

The high drug entrapments observed can be attributed to the supramolecular interactions (i.e., induced dipole interactions) between the aromatic rings in Rif and the quaternary ammonium salts in EuRS100. ${ }^{41}$ As we mentioned before, the flash evaporation of the solvent in the flight form the needle to the collector is responsible for the high drug loadings observed for the electrosprayed micro- and sub-microparticles. However, because of the slight water solubility $\left(2.5 \mathrm{mg} / \mathrm{mL}\right.$, at $25^{\circ} \mathrm{C}$ and $\mathrm{pH}$ 7.3) of the Rif, attributed to the polar groups in the molecule (two on the napthol ring and three on the ansa bridge $^{42}$ ), the loss of Rif during the aqueous nanoprecipitation process in the two miscible solvents (water and ethanol/ acetone) rendered lower drug loadings $(2.0 \pm 0.1 \mathrm{wt} \%)$. When using the electrospraying technique, flash organic solvent evaporation in the flight from the needle to the collector reduces the drug loss, whereas when using the nanoprecipitation technique, the diffusion of the organic solvent through the interface of the formed droplets is a slow process, which contributes to a partial loss of the encapsulated drug during the polymer precipitation process. Likewise, the nanoparticles obtained by nanoprecipitation are washed after synthesis to remove all the nonencapsulated drugs, and thanks to their large surface area per volume ratio, part of the drug is also lost. These reasons can explain the differences on the encapsulation efficiencies observed for the electrosprayed particles compared to the nanoparticles obtained by nanoprecipitation. Despite this reduced drug loading, the release from the nanoparticles under simulated intestinal conditions was very rapid (Figure 3), which would overcome the large limitation of reduced water solubility for Rif and its reduced bioavailability being a class II drug in the biopharmaceutics classification system. ${ }^{43}$
Another advantage of using these micro- and nanoparticulated antibiotic carriers is that the residence time in the stomach would be shorter than the one obtained from conventional solid oral dosage forms having larger sizes (e.g., tablets or capsules) and, therefore, the acidic degradation of Rif $\left(\sim 26.5 \%{ }^{9}\right)$ would be reduced. The large surface area per volume ratio available in the micro- and nanoparticles would also reach homogeneously a large surface area in the intestinal lining instead of providing with a local release from the conventional solid oral dosage forms.

Most of the previous publications in which Rif is gastroprotected aim to obtain an improved drug solubility and a fast drug release in the stomach because under acidic conditions, the drug solubility increases, being principally absorbed in the stomach and duodenum. ${ }^{43,44}$ Herein, we show that different drug release kinetics can be obtained under simulated intestinal conditions, avoiding gastric degradation.

Hydroxypropyl cellulose alone and in combination with $\mathrm{pH}$ dependent Eudragit L100-55 have been used in the fabrication of matrix tablets containing Rif and isoniazid. ${ }^{45}$ Even though these authors showed that Rif solubility was $\sim 40$ times higher under acidic conditions, Eudragit was also included in the formulation to achieve gastroprotection and to promote drug release in the intestine for up to $24 \mathrm{~h}$. However, in this case, the polymer completely dissolves at $\mathrm{pH} 5.5$ by salt formation, releasing the contained cargo completely. In our vehicle, a sustained $\mathrm{pH}$-independent drug release is achievable. A similar polymer (Eudragit RL100) was used by Ammar and Khalil ${ }^{12}$ to coencapsulate paracetamol and Rif prepared by coevaporation of the solvent containing the acrylic polymer together with the drugs. In this case, a microparticulate $(250 \mu \mathrm{m})$ mixed matrix was obtained after grinding and sieving the drug and polymer together in order to analyze the influence of the polymer in the drug dissolution rates under simulated gastric and intestinal conditions. Using this vehicle, $60 \%$ of the drug was solubilized in only $8 \mathrm{~h}$.

Several in vivo studies have demonstrated the enhanced efficacy of administering a microencapsulated drug orally in EuRS100 over the effect of the equivalent dose of the free drug. For instance, Meena et al. ${ }^{46}$ demonstrated, in chemically induced diabetic rats, that EuRS100-based microencapsulated catechin (a bioflavonoid with efficacy against type- 2 diabetes) daily administered orally for 60 days produces a superior hypoglycemic effect than the free natural compound. The same polymer has been used for the microencapsulation $(53 \mu \mathrm{m})$ of one of the low-molecular-weight heparins (i.e., nadroparin) in the oral administration using preclinical models (male adult New Zealand rabbits) being their anticoagulative effect compared with the equivalent dose of a commercial formulation of the free drug subcutaneously (SC) injected. ${ }^{47}$ 
Plasmatic anticoagulation activity was maintained at the same level than the one achieved after SC administration, but high relative bioavailabilities (up to 40\%) were achieved when using the microparticulated form.

Furthermore, the particles developed in our work would be largely excluded from reaching the systemic circulation because of their mucoadhesive nature caused by the positively charged quaternary ammonium groups in addition to the dense thickness of the mucus which varies from $\sim 123 \mu \mathrm{m}$ in the jejunum to $\sim 830 \mu \mathrm{m}$ in the colon. ${ }^{48}$ In addition, because of the intercellular tight junction, complexes present in the intestinal epithelium macromolecules above $1 \mathrm{~nm}$ are largely excluded to percolate. $^{49}$ Therefore, the microparticles would be largely excluded to be absorbed intact through the intestinal wall. In a potential envisaged application, these formulations would be orally administered as a suspension bypassing gastric degradation, attaching to the intestinal wall, eroding slowly in a time-controlled manner, and releasing their cargo.

As we mentioned before, a sustained antimicrobial action of antibiotics against infections caused by $S$. aureus is required in different scenarios including implant-associated infections, indwelling medical devices, and chronic osteomyelitis. We showed that the encapsulation in the acrylic polymers of the model antibiotic did not impair its antibacterial action (Figure 4). The prevalence of periprosthetic infection is established in $1-2 \%$ for total hip and knee arthroplasties, and in revision surgeries, the values for infected replacements increase to 5$40 \%$, whereas for fixation devices on open fractures, the infection incidence exceeds $30 \% .^{50,51}$ Because of the small size of the EuRS100 nanoparticles ( 20 nm) achievable, a burst Rif release is observed under neutral $\mathrm{pH}$, and consequently, the antimicrobial action is similar to the effect observed for the free drug (Figure 4). Nevertheless, for the submicron EuRS100 $(\sim 200 \mathrm{~nm})$ and microparticles $(\sim 2 \mu \mathrm{m})$, a superior antimicrobial effect was observed, probably caused by the combination of the mucoadhesive character of the positively charged polymer, which electrostatically interacts with the negatively charged bacterial wall, and the time-dependent sustained release achieved, promoting antibiotic intracellular transport.

The reduced cytotoxicity observed on mammalian cell lines for the different empty and Rif-loaded particles prepared in this work (Figure 5) is also corroborated in previous studies. ${ }^{52,53}$ According to the pharmacopeia, ${ }^{12}$ a daily intake of $2-200 \mathrm{mg} /$ $\mathrm{kg}$ bodyweight depending on the grade of Eudragit is considered as largely safe in humans. In addition, the lack of cytotoxicity against eukaryotic cells, also observed for Rif even at the maximum dose tested $(21 \mu \mathrm{g} / \mathrm{mL})$, is in agreement with the previous literature. ${ }^{54}$ IC50 for Rif on human fibroblasts has been reported for doses one order of magnitude higher than the ones used in this work. ${ }^{55}$ A reduced $S$ phase and increased G1 and G2 phases observed for the empty and Rif-loaded particles compared to the untreated control would be an indication of a slight cell cycle arrest. Although slight differences were observed, cellular viability was not affected at the subcytotoxic dose studied. In summary, at the doses studied, neither the acrylic polymers used nor the antibiotic induced cytotoxicity on human cells.

As we have shown, using different synthesis conditions, we are able to obtain gastroprotected antibiotic-loaded particles having tunable drug release kinetics. In some antibiotic therapy, a fast-systemic distribution is needed, whereas in other infections, a long-term antibiotic treatment is desirable.
Chronic infections (e.g., pressure or diabetic foot ulcers), recurrent urinary tract infections, chronic respiratory diseases, infections associated with intracellular pathogens (e.g., tuberculosis), implant-associated infections, and primary and secondary prophylaxis are usually managed with long-term antibiotic therapy. Fast releasing systems are necessary in acute infections, in prophylaxis (e.g., in wounds, burns, joint replacements, C-sections, etc.), and also in the management of endogenous diseases (e.g., sepsis, urinary tract infections, skin and soft tissue infections, etc.). A prolonged release would be desirable in chronic communicable diseases (e.g., tuberculosis, respiratory and healthcare bacterial infections, etc.). Herein, we have demonstrated that using different synthesis protocols, we can produce antibiotic-loaded particles that can provide with a burst or prolonged release over time and reach antimicrobial levels at different time points.

A recent study of Klein et al. ${ }^{56}$ showed that despite the preventive campaigns to spread awareness on antibiotic resistance, the antibiotic use in 76 countries studied over 16 years (2000-2015) increased by $65 \%$ (expressed as prescribed daily doses), and the antibiotic consumption rate increased by $39 \%$. Therefore, the development of controlled antibiotic releasing systems which provide the required antimicrobial concentration without underdosing or overdosing for the specific acute or chronic infections represents an advantage compared to the uncontrolled systemic therapy and provides with a rational usage of drugs.

\section{CONCLUSIONS}

Time-controlled release $\mathrm{pH}$-independent polymers have been used in the synthesis of Rif-loaded nano-, submicro-, and microparticles using electrospraying and micromixing-assisted nanoprecipitation-solvent evaporation techniques. The different particle sizes allow producing a burst or a long-term antibiotic release, reaching MICs and MBCs in the hour or day range depending on the needs. The encapsulation of the antibiotic did not impair its antimicrobial action against Gram positive bacteria. At low concentrations, the encapsulated antibiotic showed superior antimicrobial action compared to the equivalent doses of the free wide-spectrum antibiotic. The polymeric carriers and resulting antibiotic-loaded particles did not produce cytotoxic effects on human cell lines or significant cell arrests after analyzing the cell cycles. The developed materials are promising vectors to provide the correct antibiotic doses according to the type of infection.

\section{ASSOCIATED CONTENT}

\section{SI Supporting Information}

The Supporting Information is available free of charge at https://pubs.acs.org/doi/10.1021/acs.molpharmaceut.0c00408.

Morphological analysis of the particles synthesized and mathematical analysis performed to fit the drug release data to the kinetic model for the different particles (PDF)

\section{AUTHOR INFORMATION}

\section{Corresponding Authors}

Victor Sebastian - Department of Chemical Engineering, Aragon Institute of Nanoscience (INA), University of Zaragoza, Aragón Materials Science Institute, ICMA, 50018 Zaragoza, Spain; Aragon Health Research Institute (IIS Aragon), 50009 
Zaragoza, Spain; Networking Research Center on Bioengineering, Biomaterials and Nanomedicine, CIBER-BBN, 28029 Madrid, Spain; 이이.orid/0000-0002-6873-5244; Email: arruebom@unizar.es

Manuel Arruebo - Department of Chemical Engineering, Aragon Institute of Nanoscience (INA), University of Zaragoza, Aragón Materials Science Institute, ICMA, 50018 Zaragoza, Spain; Aragon Health Research Institute (IIS Aragon), 50009 Zaragoza, Spain; Networking Research Center on Bioengineering, Biomaterials and Nanomedicine, CIBER-BBN, 28029 Madrid, Spain; 이이이.org/0000-0003-3165-0156; Email: victorse@unizar.es

\section{Authors}

Cristina Yus - Department of Chemical Engineering, Aragon Institute of Nanoscience (INA), University of Zaragoza, Aragón Materials Science Institute, ICMA, 50018 Zaragoza, Spain; Aragon Health Research Institute (IIS Aragon), 50009 Zaragoza, Spain

Silvia Irusta - Department of Chemical Engineering, Aragon Institute of Nanoscience (INA), University of Zaragoza, Aragón Materials Science Institute, ICMA, 50018 Zaragoza, Spain; Aragon Health Research Institute (IIS Aragon), 50009 Zaragoza, Spain; Networking Research Center on Bioengineering, Biomaterials and Nanomedicine, CIBER-BBN, 28029 Madrid, Spain; 이이이.org/0000-0002-2966-9088

Complete contact information is available at:

https://pubs.acs.org/10.1021/acs.molpharmaceut.0c00408

\section{Author Contributions}

The manuscript was written through contributions of all authors. All authors have given approval to the final version of the manuscript.

\section{Notes}

The authors declare no competing financial interest.

\section{ACKNOWLEDGMENTS}

Funding: this research was funded by the Spanish Ministry of Economy and Competitiveness (grant number CTQ2017$84473-R)$. Financial support from the EU thanks to the ERC Consolidator Grant program (ERC-2013-CoG-614715, NANOHEDONISM) is also acknowledged. CIBER-BBN is an initiative funded by the VI National R\&D\&i Plan 20082011, Iniciativa Ingenio 2010, Consolider Program, CIBER Actions and financed by the Instituto de Salud Carlos III (Spain) with assistance from the European Regional Development Fund. V.S. acknowledges the financial support of Ministerio de Ciencia, Innovación y Universidades, Programa Retos Investigación, Proyecto REF: RTI2018-099019-A-I00.

\section{ABBREVIATIONS}

Rif, rifampicin; EuRS100, Eudragit RS100; MIC, minimum inhibitory concentration; MBC, minimum bactericidal concentration

\section{REFERENCES}

(1) Sung, J. C.; Padilla, D. J.; Garcia-Contreras, L.; VerBerkmoes, J. L.; Durbin, D.; Peloquin, C. A.; Elbert, K. J.; Hickey, A. J.; Edwards, D. A. Formulation and Pharmacokinetics of Self-Assembled Rifampicin Nanoparticle Systems for Pulmonary Delivery. Pharm. Res. 2009, 26, 1847-1855.

(2) Lueth, P.; Haughney, S. L.; Binnebose, A. M.; Mullis, A. S.; Peroutka-Bigus, N.; Narasimhan, B.; Bellaire, B. H. Nanotherapeutic
Provides Dose Sparing and Improved Antimicrobial Activity against Brucella Melitensis Infections. J. Controlled Release 2019, 294, 288297.

(3) Li, Y.; Liu, G.; Wang, X.; Hu, J.; Liu, S. Enzyme-Responsive Polymeric Vesicles for Bacterial-Strain-Selective Delivery of Antimicrobial Agents. Angew. Chem., Int. Ed. 2016, 55, 1760-1764.

(4) Hussain, S.; Joo, J.; Kang, J.; Kim, B.; Braun, G. B.; She, Z.-G.; Kim, D.; Mann, A. P.; Mölder, T.; Teesalu, T.; Carnazza, S.; Guglielmino, S.; Sailor, M. J.; Ruoslahti, E. Antibiotic-Loaded Nanoparticles Targeted to the Site of Infection Enhance Antibacterial Efficacy. Nat. Biomed. Eng. 2018, 2, 95-103.

(5) Ali, H. R.; Ali, M. R. K.; Wu, Y.; Selim, S. A.; Abdelaal, H. F. M.; Nasr, E. A.; El-Sayed, M. A. Gold Nanorods as Drug Delivery Vehicles for Rifampicin Greatly Improve the Efficacy of Combating Mycobacterium Tuberculosis with Good Biocompatibility with the Host Cells. Bioconjugate Chem. 2016, 27, 2486-2492.

(6) Kotoky, J.; Kandimalla, R.; Kalita, S.; Devi, B.; Sharma, K. K.; Sharma, A.; Kalita, K.; Kataki, A. C. Chloramphenicol Encapsulated in Poly- $\varepsilon$-Caprolactone-Pluronic Composite: Nanoparticles for Treatment of MRSA-Infected Burn Wounds. Int. J. Nanomed. 2015, 10, 2971-2984.

(7) Pereira, J. M.; Mejia-Ariza, R.; Ilevbare, G. A.; McGettigan, H. E.; Sriranganathan, N.; Taylor, L. S.; Davis, R. M.; Edgar, K. J. Interplay of Degradation, Dissolution and Stabilization of Clarithromycin and Its Amorphous Solid Dispersions. Mol. Pharm. 2013, 10, 4640-4653.

(8) Winslow, C. J.; Nichols, B. L. B.; Novo, D. C.; MosqueraGiraldo, L. I.; Taylor, L. S.; Edgar, K. J.; Neilson, A. P. CelluloseBased Amorphous Solid Dispersions Enhance Rifapentine Delivery Characteristics in Vitro. Carbohydr. Polym. 2018, 182, 149-158.

(9) Singh, H.; Bhandari, R.; Kaur, I. P. Encapsulation of Rifampicin in a Solid Lipid Nanoparticulate System to Limit Its Degradation and Interaction with Isoniazid at Acidic PH. Int. J. Pharm. 2013, 446, $106-111$.

(10) Saraogi, G. K.; Gupta, P.; Gupta, U. D.; Jain, N. K.; Agrawal, G. P. Gelatin Nanocarriers as Potential Vectors for Effective Management of Tuberculosis. Int. J. Pharm. 2010, 385, 143-149.

(11) Andreu, V.; Larrea, A.; Rodriguez-Fernandez, P.; Alfaro, S.; Gracia, B.; Lucía, A.; Usón, L.; Gomez, A.-C.; Mendoza, G.; Lacoma, A.; Dominguez, J.; Prat, C.; Sebastian, V.; Ainsa, J. A.; Arruebo, M. Matryoshka-Type Gastro-Resistant Microparticles for the Oral Treatment of Mycobacterium Tuberculosis. Nanomedicine 2019, 14, $707-726$.

(12) Ammar, H. O.; Khalil, R. M. Preparation and Evaluation of Sustained-Release Solid Dispersions of Drugs with Eudragit Polymers. Drug Dev. Ind. Pharm. 1997, 23, 1043-1054.

(13) Bayston, R.; Ashraf, W.; Pelegrin, I.; Fowkes, K.; Bienemann, A. S.; Singleton, W. G. B.; Scott, I. S. An External Ventricular Drainage Catheter Impregnated with Rifampicin, Trimethoprim and Triclosan, with Extended Activity against MDR Gram-Negative Bacteria: An in Vitro and in Vivo Study. J. Antimicrob. Chemother. 2019, 74, 29592964.

(14) Murillo, O.; Garrigós, C.; Pachón, M. E.; Euba, G.; Verdaguer, R.; Cabellos, C.; Cabo, J.; Gudiol, F.; Ariza, J. Efficacy of High Doses of Daptomycin versus Alternative Therapies against Experimental Foreign-Body Infection by Methicillin-Resistant Staphylococcus Aureus. Antimicrob. Agents Chemother. 2009, 53, 4252-4257.

(15) Sandberg, A.; Hessler, J. H. R.; Skov, R. L.; Blom, J.; FrimodtMøller, N. Intracellular Activity of Antibiotics against Staphylococcus Aureus in a Mouse Peritonitis Model. Antimicrob. Agents Chemother. 2009, 53, 1874-1883.

(16) Russell, C. D.; Lawson McLean, A.; Saunders, C.; Laurenson, I. F. Adjunctive Rifampicin May Improve Outcomes in Staphylococcus Aureus Bacteraemia: A Systematic Review. J. Med. Microbiol. 2014, 63, $841-848$.

(17) Tuchscherr, L.; Kreis, C. A.; Hoerr, V.; Flint, L.; Hachmeister, M.; Geraci, J.; Bremer-Streck, S.; Kiehntopf, M.; Medina, E.; Kribus, M.; Raschke, M.; Pletz, M.; Peters, G.; Löffler, B. Staphylococcus Aureus Develops Increased Resistance to Antibiotics by Forming 
Dynamic Small Colony Variants during Chronic Osteomyelitis. J. Antimicrob. Chemother. 2016, 71, 438-448.

(18) Jørgensen, N. P.; Skovdal, S. M.; Meyer, R. L.; Dagnæs-Hansen, F.; Fuursted, K.; Petersen, E. Rifampicin-Containing Combinations Are Superior to Combinations of Vancomycin, Linezolid and Daptomycin against Staphylococcus Aureus Biofilm Infection in Vivo and in Vitro. Pathog. Dis. 2016, 74, ftw019.

(19) Lee, C.-Y.; Huang, C.-H.; Lu, P.-L.; Ko, W.-C.; Chen, Y.-H.; Hsueh, P.-R. Role of Rifampin for the Treatment of Bacterial Infections Other than Mycobacteriosis. J. Infect. 2017, 75, 395-408.

(20) Arruebo, M.; Uson, L.; Miana, M.; Ortiz De Solorzano, I.; Sebastian, V.; Larrea, A. Continuous Synthesis of Drug-Loaded Nanoparticles Using Microchannel Emulsification and Numerical Modeling: Effect of Passive Mixing. Int. J. Nanomed. 2016, 11, 33973416.

(21) Larrea, A.; Clemente, A.; Luque-Michel, E.; Sebastian, V. Efficient Production of Hybrid Bio-Nanomaterials by Continuous Microchannel Emulsification: Dye-Doped $\mathrm{SiO} 2$ and Au-PLGA Nanoparticles. Chem. Eng. J. 2017, 316, 663-672.

(22) Chen, W.; Palazzo, A.; Hennink, W. E.; Kok, R. J. The Effect of Particle Size on Drug Loading and Release Kinetics of GefitinibLoaded PLGA Microspheres. Mol. Pharmaceutics 2016, 14, 459.

(23) Rowe, R.; Sheskey, P.; Quinn, M. Handbook of Pharmaceutical Excipients. 6th ed.; Pharmaceutical Press, 2009.

(24) Wesley, A.; Mantle, M.; Man, D.; Qureshi, R.; Forstner, G.; Forstner, J. Neutral and Acidic Species of Human Intestinal Mucin. Evidence for Different Core Peptides. J. Biol. Chem. 1985, 260, 79557959.

(25) Agrawal, S.; Ashokraj, Y.; Bharatam, P. V.; Pillai, O.; Panchagnula, R. Solid-State Characterization of Rifampicin Samples and Its Biopharmaceutic Relevance. Eur. J. Pharm. Sci. 2004, 22, 127144.

(26) Sunoqrot, S.; Abujamous, L. PH-Sensitive Polymeric Nanoparticles of Quercetin as a Potential Colon Cancer-Targeted Nanomedicine. J. Drug Delivery Sci. Technol. 2019, 52, 670-676.

(27) Schianti, J. N.; Cerize, N. N. P.; de Oliveira, A. M.; Derenzo, S.; Seabra, A. C.; Góngora-Rubio, M. R. Rifampicin Nanoprecipitation Using Flow Focusing Microfluidic Device. J. Nanomed. Nanotechnol. 2013, 4, 2 .

(28) Deshmukh, R. K.; Naik, J. B. Diclofenac Sodium-Loaded Eudragit ${ }^{\circledR}$ Microspheres: Optimization Using Statistical Experimental Design. J. Pharm. Innovation 2013, 8, 276-287.

(29) Peppas, N. A.; Sahlin, J. J. A Simple Equation for the Description of Solute Release. III. Coupling of Diffusion and Relaxation. Int. J. Pharm. 1989, 57, 169-172.

(30) Siepmann, J.; Peppas, N. A. Higuchi Equation: Derivation, Applications, Use and Misuse. Int. J. Pharm. 2011, 418, 6-12.

(31) Ritger, P. L.; Peppas, N. A. A simple equation for description of solute release I. Fickian and non-fickian release from non-swellable devices in the form of slabs, spheres, cylinders or discs. J. Controlled Release 1987, 5, 23-36.

(32) Lindner, W. D.; Lippold, B. C. Drug Release From Hydrocolloid Embeddings with High or Low Susceptibility to Hydrodynamic Stress. Pharm. Res. 1995, 12, 1781-1785.

(33) Foldes, M.; Munro, R.; Sorrell, T. C.; Shanker, S.; Toohey, M. In-Vitro Effects of Vancomycin, Rifampicin, and Fusidic Acid, Alone and in Combination, against Methicillin-Resistant Staphylococcus Aureus. J. Antimicrob. Chemother. 1983, 11, 21-26.

(34) Norden, C. W.; Shaffer, M. Treatment of Experimental Chronic Osteomyelitis Due to Staphylococcus Aureus with Vancomycin and Rifampin. J. Infect. Dis. 1983, 147, 352-357.

(35) Naha, P. C.; Byrne, H. J.; Panda, A. K. Role of Polymeric Excipients on Controlled Release Profile of Glipizide from PLGA and Eudragit RS 100 Nanoparticles. J. Nanopharmaceutics Drug Deliv. 2013, 1, 74-81.

(36) Pan, W.; Zhang, W.; Li, X.; Ye, T.; Chen, F.; Yu, S.; Chen, J.; Yang, X.; Yang, N.; Zhang, J.; Liu, J.; Kong, J. Nanostructured Lipid Carrier Surface Modified with Eudragit RS 100 and Its Potential Ophthalmic Functions. Int. J. Nanomed. 2014, 9, 4305-4315.
(37) Gracia, R.; Yus, C.; Abian, O.; Mendoza, G.; Irusta, S.; Sebastian, V.; Andreu, V.; Arruebo, M. Enzyme Structure and Function Protection from Gastrointestinal Degradation Using Enteric Coatings. Int. J. Biol. Macromol. 2018, 119. DOI: 10.1016/ j.ijbiomac.2018.07.143.

(38) Singh, M.; Sasi, P.; Rai, G.; Gupta, V. H.; Amarapurkar, D.; Wangikar, P. P. Studies on Toxicity of Antitubercular Drugs Namely Isoniazid, Rifampicin, and Pyrazinamide in an in Vitro Model of HepG2 Cell Line. Med. Chem. Res. 2011, 20, 1611-1615.

(39) Vibe, C. B.; Fenaroli, F.; Pires, D.; Wilson, S. R.; Bogoeva, V.; Kalluru, R.; Speth, M.; Anes, E.; Griffiths, G.; Hildahl, J. Thioridazine in PLGA Nanoparticles Reduces Toxicity and Improves Rifampicin Therapy against Mycobacterial Infection in Zebrafish. Nanotoxicology 2016, 10, 680-688.

(40) Isefuku, S.; Joyner, C. J.; Simpson, A. H. R. W. Toxic Effect of Rifampicin on Human Osteoblast-like Cells. J. Orthop. Res. 2001, 19, 950-954.

(41) Kim, K. S.; Lee, J. Y.; Lee, S. J.; Ha, T.-K.; Kim, D. H. On Binding Forces between Aromatic Ring and Quaternary Ammonium Compound. J. Am. Chem. Soc. 1994, 116, 7399-7400.

(42) Campbell, E. A.; Korzheva, N.; Mustaev, A.; Murakami, K.; Nair, S.; Goldfarb, A.; Darst, S. A. Structural Mechanism for Rifampicin Inhibition of Bacterial RNA Polymerase. Cell 2001, 104, 901-912.

(43) Luciani-Giacobbe, L. C.; Ramírez-Rigo, M. V.; Garro-Linck, Y.; Monti, G. A.; Manzo, R. H.; Olivera, M. E. Very Fast Dissolving Acid Carboxymethylcellulose-Rifampicin Matrix: Development and SolidState Characterization. Eur. J. Pharm. Sci. 2017, 96, 398-410.

(44) Maurya, D. P.; Sultana, Y.; Aqil, M.; Ali, A. Formulation and Optimization of Rifampicin Microparticles by Box-Behnken Statistical Design. Pharm. Dev. Technol. 2012, 17, 687-696.

(45) Hiremath, P. S.; Saha, R. N. Oral Matrix Tablet Formulations for Concomitant Controlled Release of Anti-Tubercular Drugs: Design and in Vitro Evaluations. Int. J. Pharm. 2008, 362, 118-125.

(46) Meena, K. P.; Vijayakumar, M. R.; Dwibedy, P. S. CatechinLoaded Eudragit Microparticles for the Management of Diabetes: Formulation, Characterization and in Vivo Evaluation of Antidiabetic Efficacy. J. Microencapsulation 2017, 34, 342-350.

(47) Javot, L.; Sapin, A.; Scala-Bertola, J.; Vigneron, C.; Lecompte, T.; Maincent, P. Oral Administration of a Microencapsulated LowMolecular-Weight Heparin to Rabbits: Anti-Xa and Anti-IIa Profiles. Thromb. Haemostasis 2010, 103, 1254-1267.

(48) Atuma, C.; Strugala, V.; Allen, A.; Holm, L. The Adherent Gastrointestinal Mucus Gel Layer: Thickness and Physical State in Vivo. Am. J. Physiol.: Gastrointest. Liver Physiol. 2001, 280, G922G929.

(49) Shen, L.; Weber, C. R.; Raleigh, D. R.; Yu, D.; Turner, J. R. Tight Junction Pore and Leak Pathways: A Dynamic Duo. Annu. Rev. Physiol. 2011, 73, 283-309.

(50) Widmer, A. F. New Developments in Diagnosis and Treatment of Infection in Orthopedic Implants. Clin. Infect. Dis. 2001, 33, S94S106.

(51) Trampuz, A.; Widmer, A. F. Infections Associated with Orthopedic Implants. Curr. Opin. Infect. Dis. 2006, 19, 349.

(52) Yus, C.; Gracia, R.; Larrea, A.; Andreu, V.; Irusta, S.; Sebastian, V.; Mendoza, G.; Arruebo, M. Targeted Release of Probiotics from Enteric Microparticulated Formulations. Polymers 2019, 11, 1668.

(53) Yenilmez, E.; Yurtdaş Kırımlıoğlu, G.; Şenel, B.; Basaran, E. Preparation, Characterization and in Vitro Evaluation of Dirithromycin Loaded Eudragitr® RS100 Nanoparticles for Topical Application. Lat. Am. J. Pharm. 2017, 36, 2203-2212.

(54) Panova, N.; Zborníková, E.; Šimák, O.; Pohl, R.; Kolář, M.; Bogdanová, K.; Večeřová, R.; Seydlová, G.; Fišer, R.; Hadravová, R.; Šanderová, H.; Vítovská, D.; Siková, M.; Látal, T.; Lovecká, P.; Barvík, I.; Krásný, L.; Rejman, D. Insights into the Mechanism of Action of Bactericidal Lipophosphonoxins. PLoS One 2015, 10, e0145918.

(55) Durán, N.; Alvarenga, M. A.; Da Silva, E. C.; Melo, P. S.; Marcato, P. D. Microencapsulation of Antibiotic Rifampicin in 
Poly(3-Hydroxybutyrate-Co-3-Hydroxyvalerate). Arch. Pharmacal Res. 2008, 31, 1509-1516.

(56) Klein, E. Y.; Van Boeckel, T. P.; Martinez, E. M.; Pant, S.; Gandra, S.; Levin, S. A.; Goossens, H.; Laxminarayan, R. Global Increase and Geographic Convergence in Antibiotic Consumption between 2000 and 2015. Proc. Natl. Acad. Sci. U.S.A. 2018, 115, E3463-E3470. 